\title{
STRUCTURAL HEALTH MONITORING OF BRIDGES WITH PIEZOELECTRIC
} AE SENSORS

\author{
O. Yapar ${ }^{\text {a }}$, P.K. Basu ${ }^{\mathrm{a}, *}$, P. Volgyesi ${ }^{\mathrm{b}}$, A. Ledeczi ${ }^{\mathrm{b}}$ \\ ${ }^{a}$ Department of Civil and Environmental Engineering, Vanderbilt University \\ 2201 West End Ave, Nashville, TN 37235 \\ ${ }^{b}$ Institute for Software Integrated Systems (ISIS), Vanderbilt University \\ 1025 16th Ave S, Suite 102 Nashville, TN 37212
}

\begin{abstract}
Monitoring the health of structures using acoustic emission technique offers many advantages such as early and quick detection of damage in real-time inexpensively. This paper is concerned with health monitoring of infrastructure hardware like highway bridges using this technique for timely intervention preventing catastrophic failure. Both experimental and numerical investigations were undertaken to determine the effectiveness and applicability of the method in the case of bridge superstructures. Three types of representative bridge girders of steel, reinforced concrete and prestressed concrete were designed, fabricated and monitored in the laboratory for structural integrity under cyclic loads with the help of an acoustic emission sensor system. After laboratory work, wavelet and Fourier transform techniques were applied to the recorded signals for de-noising and to diagnose the damaged state of the structure. Finally the locations of the cracks were determined by using the artificial neural network (ANN) approach.
\end{abstract}

Keywords: Acoustic emission; Bridge failures; Crack growth; Damage assessment; Nondestructive testing

\section{INTRODUCTION}

There is hardly an engineering material which is perfect in all respects and built-in imperfections of various sorts cannot be avoided. As such materials are stressed, the imperfections within the material tend to grow in the form of cracks, slips, dislocations, etc. which may eventually reach dangerous proportions leading to failure. Growth of imperfections is accompanied by the release of short bursts of acoustic energy called acoustic emissions. Such release of energy causes stress waves travelling in the body of the structure and can be detected with special piezoelectric receivers called acoustic emission (AE) sensors, strategically mounted on the structure. Through appropriate processing of the captured signals from one or more sensors based on their time of arrival, intensity and rate, it

*Corresponding author. tel.: +1-615-322-7477; fax. +1-615-322-3365.

E-mail address: prodyot.k.basu@vanderbilt.edu (P.K.Basu). 
should be possible to fully specify the damage location and, possibly, the nature and extent of damage caused by the stresses driving it.

The first application of AE technique was to Polaris missile chambers, which was almost 50 years ago, during hydro-testing of the structure [1]. Since then the reported application of this technique in the literature has been increasing steadily. Although AE technique has been successfully applied to engineering structures in many industries including aerospace, nuclear, medical, petroleum and manufacturing, the focus of the current research effort will be on its use to monitor the health of bridge structures which are an important component of the transportation infrastructure requiring timely intervention against structural distress causing failure.

The primary aim of this paper is to investigate the efficiency and feasibility of the application of $\mathrm{AE}$ technique in ensuring structural integrity of bridge superstructures by health monitoring. In this study, prestressed concrete, reinforced concrete and steel girders are used as representative structural elements, because of widespread use in bridge construction. After the representative specimens are designed, fabricated and tested, mostly, under cyclic loading, the captured stress wave data as electrical signals by the acoustic emission system are processed for diagnostic evaluation. The background (or white) noises are first filtered out by wavelet transformation. Thereafter, power spectrums of the residual signals are created by Fourier transformation, allowing classification of the signals originating from cracks and other damage sources. By training a neural network with simulated acoustic emission events, the source location of the acoustic emission caused by crack growth is determined.

During an acoustic emission testing, all diagnostic evaluations are done by analyzing the features of the waveforms captured by the piezoelectric sensors. A typical acoustic emission burst signal usually rises first to maximum amplitude and then dampens out rapidly. An idealized acoustic emission signal is shown in Fig. 1. This signal can be represented by Eq. 1 [2]. In this equation, the notations $V, V_{0}, B, t$ and $\omega$ are output voltage, initial signal amplitude, decay constant, time and angular frequency, respectively.

$$
V=V_{0} \cdot e^{(-B . t) \cdot \sin \omega t}
$$

Although acoustic emission signal characterization can provide important information about the source, material and structure under test, identifying the original signal generated by the source is a highly complex problem. According to majority of publications on the topic, the determination of unmodified waveform and frequency spectrum of the AE signal itself is considered as intractable due to the following reasons [3]:

- The amplitude and frequency response characteristics of conventional AE sensors tend to modify the captured signals at or near the resonant frequency of the sensors.

- As the material of the coupling agent between the sensor and the specimen is different from that of the test structure, the measured signals may get physically modified. 
- Out of factory performance characteristics of AE sensors usually change or degrade with time causing uncertainties in the recorded signals.

- During the propagation of the signal in the structure under test, the generated wave gets modified due to diffusion, reflection and refraction according to the acoustical transfer characteristics of the specimen.

In spite of such bleak outlook on the part of a section of researchers, a number of others have seriously attempted to overcome the stated shortcomings. For instance, in the study by Hsu et al. a numerical method has been put forward to compute the displacement as a function of time at an arbitrary point in an infinite plate [4]. The results generated by using the method are then compared with the experimental data obtained by applying a point force as a delta function on the plate. The agreement between the data gathered by a capacitive transducer and theory was excellent. However, when the capacitive transducer was replaced by a commercial piezoelectric AE sensor, only qualitative agreement could be achieved between the sensor voltage output and the time derivative of the displacement data generated by theory.

Although, as previously shown, the developed theory can be verified with a capacitive transducer for simple geometries like a large plate, but as the geometry of the specimen assumed more complex forms, the waveform and its frequency tended to undergo significant modification due to different paths followed by the Rayleigh wave travelling parallel to the surface and the compression or body waves undergoing repeated reflections at the free surfaces. Due to such effects, the recorded signals get distorted. Schematic illustrations of this aspect for two simple geometries are shown in Fig. 2.

Weisinger proposed a theoretical method to predict the particle displacements inside and on the surface of a specimen with known material properties and geometry [5]. In this publication, only exact solution for a half-space and normal mode solution of a sphere are provided. According to the results of the study, time-displacement records of the elastic waves for spherical geometries can easily be obtained. In spite of the success of previous research work in predicting the time-displacement histories of acoustic waves, it is not always possible to obtain closed form solution to identify particle motions for any arbitrary geometry. Especially the edges of solids with complex geometry introduce unique boundary conditions lead to extremely complex mathematical equations. In this situation, the best approach to solve such complex cases is to resort to the finite element method.

In the published literature, several case studies have been reported to model wave propagation problems in different disciplines like aerospace industry [6] [7], geotechnics [8] and earthquake engineering [9] by using the finite element method. Good agreement between finite element analysis results and experimental data signified that when the model is set up right, the finite element method can be a very powerful and reliable tool to solve complex wave propagation problems and for the validation of experimental data.

Another approach followed by researchers to diagnose the damage state of structures without directly dealing with waveforms and the associated complexities, is to correlate the 
secondary acoustic emission data (namely, hits) with stress intensity factor, mobile dislocation density, and plastic strain [10] [11] [12]. Although this approach worked in some cases, it failed to give a reliable correlation all the time. In the study by Dunegan and Harris, acoustic emission count rate is attempted to be correlated with Gilman's mobile dislocation model as a function of strain. As can be seen in Fig. 3a, experimental data which is represented as dashed line show excellent agreement with the theory for a specimen made out of 7075-T6 aluminum. On the other hand for an iron-3\% specimen, the theory and experimental results are found to be significantly different, as can be seen in Fig. 3b.

Although agreement between the theory and experimental data in the work of Dunegan and Harris may probably have happened by chance, in most of the re-publications of this figure, this aspect has not been pointed out. Also in several publications, necessary precautions were not taken to eliminate the extrenious noise. Additionally, in most cases, the recorded acoustic signals were assumed to be originating from the flaw without real evidence [3].

\section{METHODOLOGY}

In the present study, the possibility of successful AE sensor based health monitoring of bridges, to guard against unexpected failure, using powerful tools like wavelet transformation, power spectrums, fast Fourier transformation and neural networks is investigated. These tools are being used for the elimination of unwanted signals from the records, to cleanse out the background noise and eventually detect the source of damage.

The use of Wavelets in signal analysis is a natural extension of Fourier analysis. In order to separate the components of tangled signals one can use windowed Fourier analysis with limited success. On the other hand, the use of wavelets instead allows clear identification of all the components with high and low frequencies and that is why wavelets are sometimes termed as 'mathematical microscope' [13]. Wavelets allow conversion of the information in a signal into numbers and coefficients which can be worked on, transmitted, stored, analysed, or used to reconstruct the original signal by adding together wavelets of various sizes at different locations. The method followed to compute the coefficients is the same as in Fourier analysis, that is, the signal and the function being analysed are multiplied together, and then the integral of the product is computed. Changing of frequency can be achieved simply by squeezing or stretching the chosen wavelet. In short, wavelets are mathematical functions of limited duration with zero average value; whereas, wavelet transformation is the breakdown of the original signal into scaled and shifted versions of the original wavelet. The main advantage of using wavelets is not to ignore the local details in the signal unlike traditional signal analysis methods. Consequently, the noisy components in the signal can be identified with minimum degradation of the original signal of interest.

After the recorded data is cleaned of the spurious noise, the actual signal generated by the wave originating from the actual source of interest can be identified. Signals generated by crack growth have much different frequency components than signals generated by other types of sources [2]. Therefore, after the signals are converted from time domain into the frequency domain, source characterization of the signals can be done easily. The best way to 
do this is to identify the amount of information (say, power) contained in different frequencies of the signal. The most effective way to acquire and visualize this information is, therefore, to generate power spectrum of the signal, which describes the distribution of the power of the signal over frequency, by using fast Fourier transform. One alternative to fast Fourier transform to extract frequency data from the signal is to use continuous wavelet transformation (CWT). In Fourier transformation, local details of the signal are ignored and it is harder to visualise the frequency components of the signals. In the present study, for source characterization of the signals will be tried using both the methods.

After de-noising the signal it is necessary to determine the source of the acoustic signal. Classical algorithms for detecting source location, namely, those based on signal arrival time differences between pairs of sensors, may give acceptable results in relatively simple situations only. The classical method can, however, fail completely in the presence of dispersion, significant background noise, and material non-homogeneity. Excessive distortion of AE signals during its propagation from the source to the sensor renders the accurate detection of signal arrival time highly uncertain. In order to overcome this difficulty, a new method based on Artificial Neural Networks (ANN) is proposed to be used in this study. It is based on the use of the common AE signal parameters such as arrival time, rise time, maximum signal amplitude, signal energy, signal count, frequency and duration. By using the data sets generated by combining these parameters obtained from different training points, an ANN can be trained covering the zone of interest and used for the detection of source location of AE signals recorded during an actual event.

\section{EXPERIMENTAL PROCEDURE}

\subsection{Specimens}

Since the most widely used materials for bridge construction are steel, reinforced concrete and prestressed concrete, model bridge girders of these materials are used in this study.

Reinforced concrete beam: The beam was so proportioned that damage can be induced easily by applying a harmonic excitation with an electro-dynamic shaker within a reasonable excitation time. Cross-sectional details of the reinforced concrete beam used in the study are shown in Fig. 4. At the center of the beam there is a $6 \mathrm{~mm}$ diameter longitudinal steel rod. The length of the beam is $2.286 \mathrm{~m}$. After conducting concrete compression and split cylinder tests on concrete cylinder specimens, the compressive strength and split tensile strength of concrete have been found as $35.17 \mathrm{MPa}$ and $3.17 \mathrm{MPa}$ respectively. The yield strength of the reinforcing rod is $413.8 \mathrm{MPa}$.

Prestressed concrete beam: The details of the prestressed concrete test beam are as shown in Fig. 5. This beam was again so proportioned that damage can be easily induced with available equipment. In the bottom there are two $12.7 \mathrm{~mm}$ dia. prestressing strands with $1.862 \mathrm{GPa}$ ultimate tensile strength and in the top there are two hanger bars of $10 \mathrm{~mm}$ size. The total length of the beam is $2.591 \mathrm{~m}$ and the size of the stirrups is $6 \mathrm{~mm}$. Yield strengths of 
top reinforcement bars and stirrups are $413.8 \mathrm{MPa}$. The average concrete compressive strength of the beam at 28 -days is $4.8 \mathrm{MPa}$.

Steel beam: The test steel beam used in the study is a standard S3x5.7 section of ASTM A36 material with yield strength of $248.2 \mathrm{MPa}$. The total length of the beam is $3.35 \mathrm{~m}$. AISC section properties of S3x5.7 is shown in Table-1 [15].

\subsection{Test Setup}

Sensors and data acquisition system: For capturing the acoustic emission data, MISTRAS R15- $\alpha$ type sensor along with MISTRAS 1283 USB AE node data acquisition system is used. Signal processing of the acoustic emission data is done by using the AEWin software meant for use with the acoustic emission sensors. A R15- $\alpha$ type sensor is shown in Fig. 6. Specifications of the sensor are given in Table-2.

During cyclic excitation, as the threshold of $40 \mathrm{~dB}$ is exceeded, the data acquisition system is triggered to record an acoustic emission hit at the sampling rate of 5mbps. On the detection of a hit a waveform of $1792 \mu$ s duration is recorded, $100 \mu$ s of which precedes the trigger. The recommended values of peak definition time (PDT), hit definition time (HDT) and hit lockout time (HLT) given for each material in the equipment manual are used. Proper definition of PDT, HDT and HLT ensures the correct identification of signal peaks, reports each signal as one and only one hit and helps avoid the measurement errors during signal decay with increased data acquisition speed.

Loading systems: During the testing of reinforced concrete and steel beams, Bruel\&Kjaer Model LDS V651 electrodynamics shaker was used. PA 1000L amplifier was used to drive the shaker with a LDS FPS 10L field power supply. The loading configuration comprising of the supports and the centrally placed loading fixture linked to the shaker head, as shown in Fig. 7, was custom designed and fabricated. Table-3 presents the performance parameters of the shaker system.

As stated earlier, the prestressed concrete beam was not subjected to dynamic excitation and was tested on a different test bed using quasi-static loading instead. The reason for doing so is that the weight of the prestressed concrete beam is too much for the shaker to be able to handle it. The quai-static loading configuration of the prestressed concrete beam and the test setup as a whole can be seen in Fig. 8.

\section{TESTING}

Steel, reinforced concrete and prestressed concrete beams, were tested. The response of each beam was monitored by installing four strain gages (two at quarter points, one at top center and one at bottom center), an accelerometer and four acoustic emission sensors.

Because of similarity with the characteristics of generated acoustic signals in solids during the formation of a structural crack, pencil lead break testing is a preferred method for 
calibrating and evaluating the efficiency of an acoustic emission health monitoring system. So, before dynamic excitation, a set of pencil lead break tests were conducted on each undamaged specimen. The generated data was then studied to get a better insight into the accuracy of the system, and the nature of the stress waves generated in the medium. In addition, this data was used to train the artificial neural network for acoustic emission source location.

\subsection{Reinforced Concrete Beam}

The chosen station locations on the reinforced concrete beam for pencil lead break test are shown in Fig. 9. In the pencil lead break test process, during the forward pass, pencil leads were broken on top of the beam, from station-1 thru station-14, and during backward pass from station-14 thru station-1. These routines were repeated several times. In each pair of such routines 27 lead break signals were generated.

After undertaking the pencil lead break tests, the shaker was used to subject the reinforced concrete beam with five different loading cycles of increasing amplitudes and varying time duration. The loading cycles were of sinusoidal wave form with a frequency of $10 \mathrm{~Hz}$. The displacement amplitudes during cyclic load application were $0.254 \mathrm{~mm}, 0.508 \mathrm{~mm}, 1.270 \mathrm{~mm}$, $2.540 \mathrm{~mm}$ and $3.810 \mathrm{~mm}$. The smaller amplitudes used in first two applications were specifically chosen to enable recording the signals before crack like damages developed in the beam. In the process, the recorded signals were caused by background events like environmental noise, rubbing between the beam and supports, friction between the concrete and reinforcement, etc. However, during these two load cycles, the recorded signals were found to be devoid of sign of the existence of any such major event. Excitation with the larger amplitude of $1.270 \mathrm{~mm}$, a crack appeared in the bottom of the beam at a distance of $190.5 \mathrm{~mm}$ from the center of shaker link. On increasing the amplitude to $2.540 \mathrm{~mm}$, the crack extended to the full depth of the beam. However, no further major events were observed when the amplitude was further increased to $3.810 \mathrm{~mm}$. A general view of the test setup, showing the close-up view of the crack and locations of the accelerometer, gages and sensors can be seen in Figs. 10 to 12.

\subsection{Prestressed Concrete Beam}

This beam was subjected to two quasi-static loading cycles. The first loading cycle was for the virgin beam. The second one was for the same beam after the application of composite patch repair of the damages caused during the first stage of loading, to get an idea about the effect of patch repair on the nature of captured AE signals. During the first loading stage, when the beam was loaded up to 11.4 tons, shear cracks appeared on both sides of centerline. During this stage of loading, only two AE sensors were active. Furthermore, one of the shear cracks appeared at the location of one of these sensors (designated as channel-1) knocking it

off due to debonding. A view of the general test setup, close-up view of the cracks and locations of the gages, accelerometer and sensors are shown in Figs. 13 and 14. 
In this case also several sets of pencil lead break tests, from station- 1 in the top of the beam up to station-28 on the side, with locations as shown in Fig. 15. In each of the several repeated sets, the total number of recorded lead break signals was 28 . In the second stage, the load was applied after the initial cracks were repaired with localized bonded composite patches, details of which are reported elsewhere [16]. During this cycle of loading, the load on the beam was increased until failure. The nature of failure of the repaired beam was shearcompressive with one of the repaired cracks from the first loading cycle grew further extending from the load application point to the support roller. During this loading stage, however, all four acoustic emission sensors were operational. Location of the cracks, gages, accelerometer and sensors for this case are shown in Fig. 16.

\subsection{Steel Beam}

The support and load configuration of the steel beam and the location of four AE sensors, before undertaking the pencil lead break test at station locations 1 thru 24, are shown in Fig.17. In each of the repeated test sets, 47 signals were recorded. Before applying the cyclic load to cause damage, the undamaged beam was subjected to sinusoidal excitation with a frequency of $10 \mathrm{~Hz}$ and amplitude of $6.35 \mathrm{~mm}$. During this loading phase, several major events were recorded caused by sources other than damage initiation or growth of cracks. The data generated in this phase was later used to understand the nature of the signals generated by non-crack related sources. In order to expedite the damaging process resulting from cyclic excitation, a crack was intentionally introduced in the bottom half of the beam section at the middle of the beam with the help of hex and jeweller's saws. In the process, the time intensive process of crack initiation and growth processes was obviated. The presence of the initial crack enabled the subsequent crack growth process to occur with fewer loading cycles. The test was undertaken with three different loading cycles of increasing amplitudes over different time frames. In these three $10 \mathrm{~Hz}$ sinusoidal loading cycles, the displacement amplitudes were set at $5.08 \mathrm{~mm}, 6.35 \mathrm{~mm}$ and $12.70 \mathrm{~mm}$. The artificially generated original crack continued to grow with increasing loading cycles. The resulting stress wave signals were recorded by acoustic emission measurement system. The general test setup, close-up view of the crack and locations of the gages, accelerometer and sensors are shown in Figs. 18 to 20 .

\section{PROCESSING OF RECORDED AE SIGNALS}

During acoustic emission testing, the most challenging problem is the proper interpretation of the recorded signals to accurately identify the damage location, after stripping the recorded signals of the background noises from various sources. In this study, a powerful arsenal of mathematical tools like wavelet transformation, fast Fourier transformation, power spectrum analysis and artificial neural networks were used to overcome the problems related to noise elimination, prediction of source location and source characterization. 


\subsection{Noise elimination}

Pollution of recorded signals by background noise is an integral part of acoustic emission testing and filtering it out is essential before any sensible diagnosis can be attempted. During acoustic emission testing, sources of noise may either be external or internal to the test specimen like crack breathing, friction, impact, ambient noise etc. [2]. Various traditional signal cleansing techniques can be used to filter out the original signal or components of the original signal along with the noise. One of the most widely used methods for noise elimination during acoustic emission testing is to use band-pass filters. As the frequency of the pass band of the filter becomes narrower, most of the noise diminishes at the cost of inhibiting acoustic emission signal detection. As wavelet analysis is the most potent tool for de-noising acoustic emission signals, it is chosen here for the elimination of the background noise of the recorded signals [17].

Mathematically, a noisy signal can be represented by Eq. 2 [17] where $s_{n}, s_{o}, s, t$ and $\sigma$ represent the noise, original signal independent of the noise, recorded signal including noise, time and noise level, respectively.

$$
s(t)=s_{O}(t)+\sigma \cdot s_{n}(t)
$$

As shown in Fig. 21, de-noising of the recorded acoustic emission signal corrupted by nonstationary noise can be accomplished in three steps [17]:

1. The coefficients of noise function $s_{n}(t)$ are computed by wavelet decomposition using a mother wavelet and the level of decomposition.

2. For each decomposition level, thresholding (hard or soft) is applied to the detail coefficients.

3. By using the wavelet reconstruction technique, the signal is reconstituted based on the original approximation coefficients and the modified detail coefficients.

Decomposition is normally started by applying the mother wavelet at the smallest scale and is repeated by doubling (or, expanding) the time scale. This process is continued for a selected number of times. Hard thresholding, which tends to create discontinuities in the signal, can be described as the process of eliminating the components of the signal whose absolute values fall below a specified threshold. On the other hand, in soft thresholding, which does not create discontinuities, the absolutes values lower than the threshold are first set to zero and then the nonzero coefficients are shrunk towards zero. In this study, the mother wavelet is chosen as the symmetrical wavelet Symlets 8 (Sym8). This family of wavelets is a modified version of Daubechies wavelets with increased symmetry. The use of Sym8, shown in Fig. 22, is guided by past successes in similar situations. The decomposition level is chosen as 5 and threshold used is soft. Of the many proposed methods for setting the threshold the "Universal Threshold" method [18] is chosen. This method ensures with a high degree certainty that every sample in the wavelet transform in which the underlying function 
is exactly zero will be estimated as zero. The AE signals from crack initiation/growth, both as raw records and after de-noising, for each specimen is shown in Figs. 24 to 26.

\subsection{AE Source Location}

Determining the source of an acoustic emission signal is a highly nonlinear problem due to several factors like the degree of shape complexity of specimen, presence of nonhomogeneities in the material, sensor and equipment limitations, the effect of sensor coupling agent, and the like. Spall et al. represented this problem with Eq. 3 which is the effect of all acoustic emission events in the system on the output of a sensor located at $r$, at time $t$ [19].

$$
v(t)=\int_{0}^{t} \int_{\text {structure }} h(r, x, t-\tau) \beta(x, \tau) d x d \tau
$$

Where $v(t), x, \beta, h$ and $\tau$ are sensor output, source location, distribution of dilatation caused by acoustic emission event as a function of position and time, structural and sensor impulse response and time, respectively. Although Eq. 3 looks simple, determining $h$ and $\beta$ is extremely involved and difficult.

This study uses the Artificial Neural Network (ANN) method. Neural networks are nonlinear functional approximations of complex problems. Here, the neural network chosen is a two layer feed-forward network with sigmoid hidden neurons. The network was trained with Leverberg-Marquardt back propagation algorithm [20] also known as the damped least squares method. Data recorded from the pencil lead break tests was used to train the network and the source location was determined using the actual test data. Structure of a simple feedforward neural network is shown in Fig. 23. Back propagation learning algorithm of an ANN, like the one used in this study, can be divided into three main stages:

i. Forward propagation of the inputs through the neural network to generate the outputs.

ii. Backward propagation of the outputs through the neural network using training pattern target to calculate the deltas of the output and hidden neurons.

iii. Calculation of the weight of each synapse (connection) by multiplying its input activation with output delta.

During the training of the ANN, there were total 28 input nodes, which were the arrival time, frequency, rise time, amplitude, signal energy, count and duration for each sensor, respectively. Number of nodes used in the hidden layer was 10 . There was only one output training node which is the location of the $\mathrm{AE}$ (pencil lead break) event. Thereafter, by introducing the signal arrival times and respective signal parameters of the real acoustic signals generated by cracks as an input to the trained ANN, source location of the signals of interest were predicted. 


\subsection{AE Source Characterization}

As previously mentioned, there were no significant acoustic emission events recorded during the testing of the reinforced concrete and prestressed concrete beams before the formation of the crack. However, there were several acoustic signals received by the sensors during the testing of the steel beam before the introduction of the artificial crack and subsequent cyclic loading. A careful examination of the arrival times of the signals indicated that the signals recorded for the steel beam before the introduction of the artificial crack were most likely originating from the support elements, outside the confines of the specimen.

The most probable cause for these events appeared to be reversible rubbing between the specimen and the supports, rubbing between support components and the friction between the support nuts, bolts and plates. However, after the introduction of the artificial crack, no signals were recorded from the supports possibly because of significantly reduced stiffness of the steel beam and redistribution of stresses.

The nature of the signals coming from the supports was found to have much lower frequency than the signals originating from the crack. For further investigation, signals of interest were transformed from time-domain to frequency-domain. The most effective way to accomplish this is to generate power spectrums of the signals. By using the fast Fourier transform algorithm, discrete Fourier transformation of the signals were carried out followed by conversion into a power spectrum. Like wavelet transformation, fast Fourier transform separates the components of tangled signals. But the difference between wavelet decomposition and fast Fourier transformation depends on the way these signal components are represented. Wavelet transformation represents these components as mathematical functions of limited duration with zero average value. On the other hand, fast Fourier transformation uses sinusoidal functions. Although, as previously mentioned, continuous wavelet transformation perform much better than Fourier transforms during time-frequency analysis, in this study even fast Fourier transformation was found to be efficient for source characterization of acoustic signals, despite the stated disadvantages. This may be attributed mainly due to significant dissimilarities between the signals generated by different sources.

\section{DISCUSSION OF RESULTS}

\subsection{De-noising of Signals:}

The resemblance between theoretical acoustic signal presented in Fig. 1 and the de-noised signals, especially the ones from the steel specimen, are striking proving the success of the de-noising procedure used. Most likely, due to the non-homogenous character of concrete material as well as the presence of steel rods and strands, the signals recorded for reinforced concrete and prestressed concrete specimens experienced significant modifications during propagation inside the specimen. By using Eq. 1 and choosing $V_{0}, B$ and $\omega$ as 1,95 and $3.03 \mathrm{E}+5$, respectively, a theoretical signal is generated. Comparison of this generated signal with the de-noised signal for the steel specimen is shown in Fig.27. Agreement between theory and experiment is striking. 


\subsection{AE Source Locations:}

Data recorded during the pencil lead break and actual quasi-static load testing of the prestressed concrete beam was not found to be suitable for determining acoustic emission source location. Most of the recorded acoustic emission events were not captured by more than two sensors which made it impossible to detect the signal source. This problem can be attributed to the large size of the test specimen signifying that four sensors are not enough for an acoustic emission system for a girder of size and shape of the prestressed concrete beam. Comparisons of some of the actual damage locations with the predicted source locations for steel and reinforced concrete beam specimen are given in Tables 4 and 5. As can be seen from the results, the crack location was detected with good accuracy by using the proposed scheme.

\subsection{AE Source Identification:}

By following the described methods, power spectrums and continuous wavelet transformations of two sample signals recorded from the steel beam were generated. In Fig. 28 , time-domain plots of the signals are presented. Power spectrums and continuous wavelet transformation plots of the first sample signal which originated from the supports is shown in Figs. 29 and 31 and the second one which originated due to crack growth is shown in Figs. 30 and 32 , respectively.

After a rough comparison of the power spectrums, one can easily notice that crack related signals have higher frequency components than the noise related signals. By using the frequency difference, signals generated by rubbing/friction can easily be separated from the signal generated by crack growth in steel beam.

The same conclusion can easily be drawn from the continuous wavelet transformation plots as well. In Figs. 31 and 32, abscissae are the time, and ordinate is the "stretching scale" of the wavelets; whereas, the colors represent the "correlation scale" of the wavelets. As the number of the "stretching scale" gets higher, the wavelet which represents that part of the signal becomes more stretched. Since the rubbing/friction related signal correlates with wavelets with higher stretching scales, it can easily be concluded that crack related signals have lower frequencies than rubbing/friction related signals.

\section{CONCLUSIONS}

The effectiveness and applicability of the acoustic emission technique for monitoring the structural integrity of bridge structures as an advance warning system against possible failure are examined. Important issues related to proper application of the $\mathrm{AE}$ technique to bridge structures are expounded. Successful processing and interpretation of AE signals was achieved by using

- Noise elimination by using wavelet transformation (Section 6.1). 
- Event source location by using neural networks (Section 6.2).

- Source characterization by using both Fast Fourier and Wavelet transformations (Section 6.3).

Three model bridge girders each with typically different material type (prestressed concrete, reinforced concrete and steel) were fabricated and tested in the laboratory. The recorded data were then processed successfully by using the tools mentioned above leading to noise elimination, event source location and source characterization. The pitfalls of the methods reported in the literature, like improper filtering of background and external noise and skipping the process of validation of the sources of the signals have successfully been resolved. Waveforms recorded during the experimental work also show good agreement with theory (Fig. 27).

Although the method has successfully been applied and tested, several improvements can be made, especially, to the source location methodology. Four sensors were barely enough for source location in reinforced concrete and steel specimen with low volume, height and width. In the case of prestressed concrete specimen, even four sensors could not pick all the signals because of the size of the specimen and signal attenuation. For small sized bridge members like angles, eye bars, cables etc., source location with a fewer number of sensors (4 or less) is probably adequate. However, for a truly three-dimensional configurations, say a large bridge component like a prestressed concrete girder, a network of more (or, even, a cluster of) sensors may be needed.

\section{ACKNOWLEDGEMENTS}

This research work was supported by National Science Foundation (NSF) Grant \#1035627, "Self-Sustaining CPS for Structural Monitoring".

\section{REFERENCES}

[1] A. Green, C. Lockman and R. Steele, "Acoustic verification of structural integrity of polaris chambers," Modern plastics, vol. 41, no. 11, pp. 137-139, 1964.

[2] P. Moore, R. Miller and H. R.K., Nondestructive testing handbook, Volume 6, Acoustic emission testing, American Society for Nondestructive Testing, 2005.

[3] R. Green Jr, "Basic wave analysis of acoustic emission," in Mechanics of nondestructive testing, Springer US, 1980, pp. 55-76.

[4] N. Hsu, S. J.A. and H. S.C., "An approach to acoustic emission signal analysis-theory and experiment," Materials and evaluation, vol. 35, pp. 100-106, 1977. 
[5] R. Weisinger, "Determination of fundamental acoustic emission signal characteristis," in Mechanics of nondestructive testing, Springer US, 1980, pp. 165-185.

[6] C. Yang, L. Ye, Z. Su and M. Bannister, "Some aspects of numerical simulation for Lamb wave propagation in composite laminates," Composite structures, vol. 75, no. 1, pp. 267-275, 2006.

[7] D. Samaratunga and R. Jha, "Lamb wave propagation simulation in smart composite structures," in Proceedings of the Simulia Customer Conference, Providence, RI, 2012.

[8] R. Motamed, K. Itoh, S. Hirose, A. Takahashi and O. Kusakabe, "Evaluation of wave barriers on ground vibration reduction through numerical modeling in ABAQUS," in Proceedings of SIMULIA Customer Conference, London, UK, 2009.

[9] L. Hall, "Simulations and analyses of train-induced ground vibrations in finite element models," Soil Dynamics and Earthquake Engineering, vol. 23, no. 5, pp. 403-413, 2003.

[10] N. Nemati, B. Metrovich and A. Nanni, "Fatigue and fracture assessment of cracks in steel elements using acoustic emission," Society of Photo-Optical Instrumentation Engineers (SPIE) Conference Series, vol. 7984, p. 72, 2011.

[11] H. Dunegan and D. Harris, "Acoustic emission-a new nondestructive testing tool," Ultrasonics, vol. 7, no. 3, pp. 160-166, 1969.

[12] H. Dunegan, D. Harris and C. Tatro, "Fracture analysis by use of acoustic emission," Engineering fracture mechanics, vol. 1, no. 1, pp. 105-122, 1968.

[13] B. Burke, "The mathematical microscope: Waves, wavelets, and beyond," in A positron name Priscilla: Scientific discovery at the Fronteir, National Academies Press, 1994, pp. 196-235.

[14] O. Yapar, P. Basu, W. Seger, J. McNutt and N. Nordendale, "Local Repair of Precast Concrete Beams Using Bonded Composite Patch," in Proceedings of the prestressed concrete national bridge conference, Nashville, TN, 2012.

[15] AISC, Steel Construction Manual, 14th Edition, American Institute of Steel Construction, 2010.

[16] O. Yapar, AE Based Health Monitoring and Bonded FRP Patch Repair in Bridge Management, Nashville, TN: Dissertation. Vanderbilt University, 2015.

[17] S. Rao and B. Subramanyam, "Analysis of Acoustic Emission Signals using Wavelet Transformation Technique," Defence science journal, vol. 58, no. 4, 2008.

[18] D. Donoho and J. Johnstone, "Ideal spatial adaptation by wavelet shrinkage," Biometrika, vol. 
81, no. 3, pp. 425-455, 1994.

[19] J. Spall, J. Maryak and M. Asher, "Neural network approach to locating acoustic emission sources in non-destructive evaluation," Journal of sound and vibration, vol. 211, no. 1, pp. 133143, 1998.

[20] K. Levenberg, "A Method for the Solution of Certain Non-Linear Problems in Least Squares."," Quarterly of applied mathematics, vol. 2, pp. 164-168, 1944. 


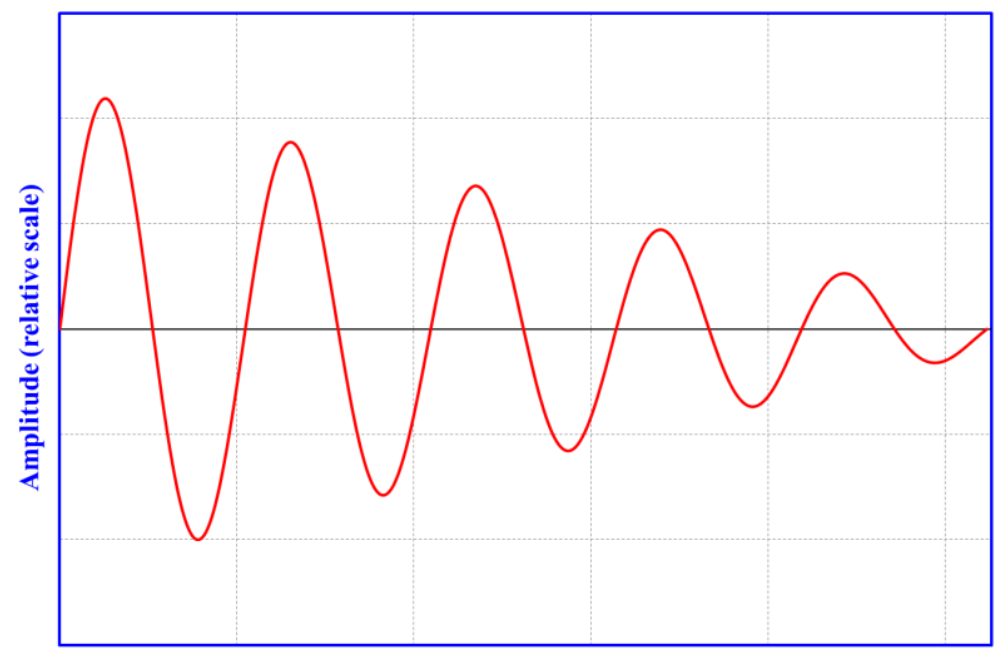

Time (relative scale)

Fig.1. Ideal AE burst signal. 


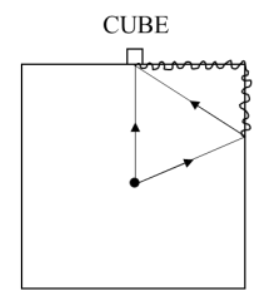

SPHERE

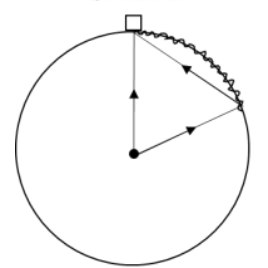

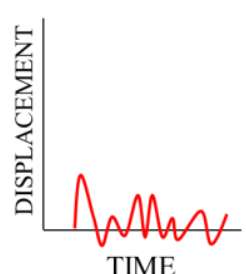

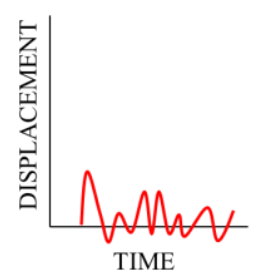

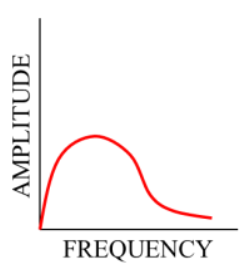

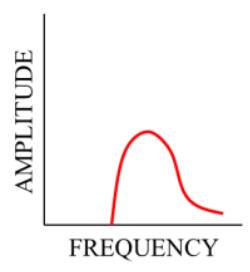

Fig. 2. Waveform and frequency spectrum modification of an acoustic signal caused by specimen geometry [3]. 


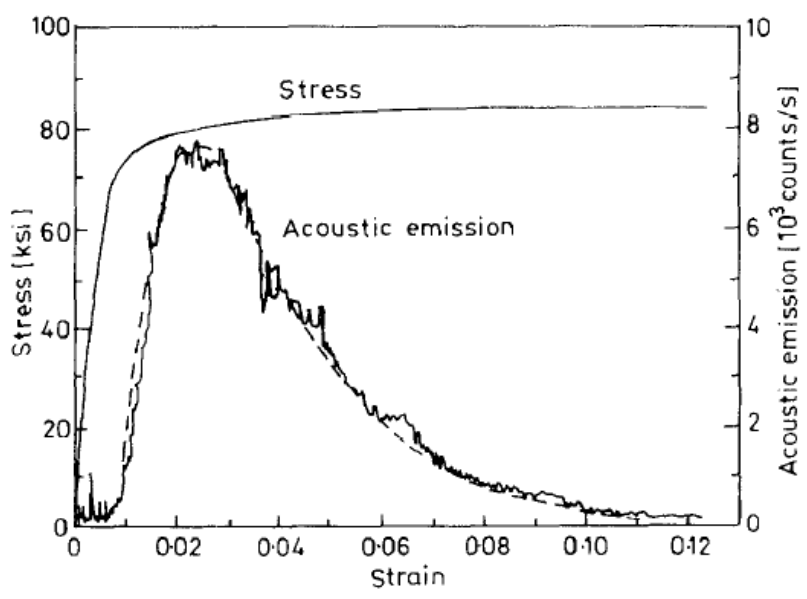

(a) 7075-T6 aluminum

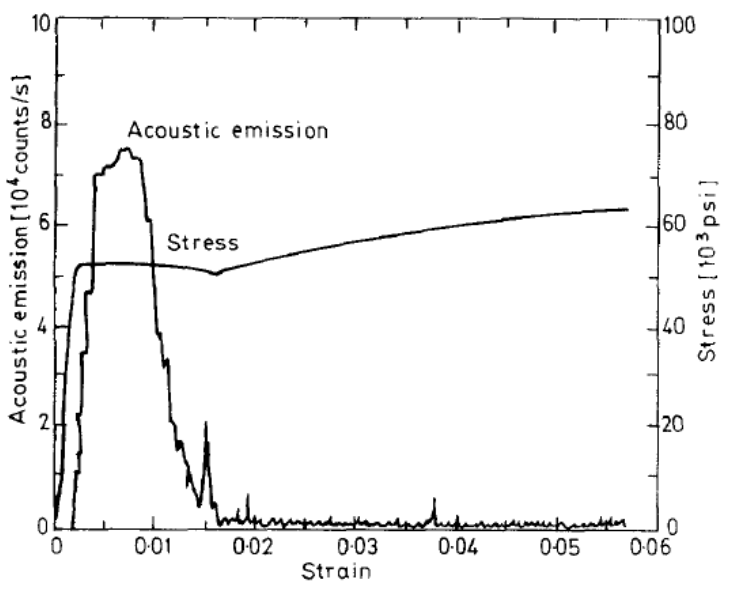

(b) Iron-3\%

Fig.3. Acoustic emission rate and stress as a function of strain [11]. 


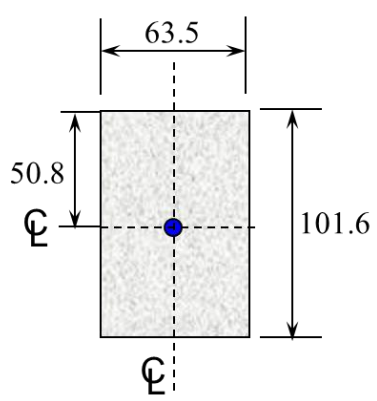

Fig.4. Cross-section of reinforced concrete test beam (mm). 


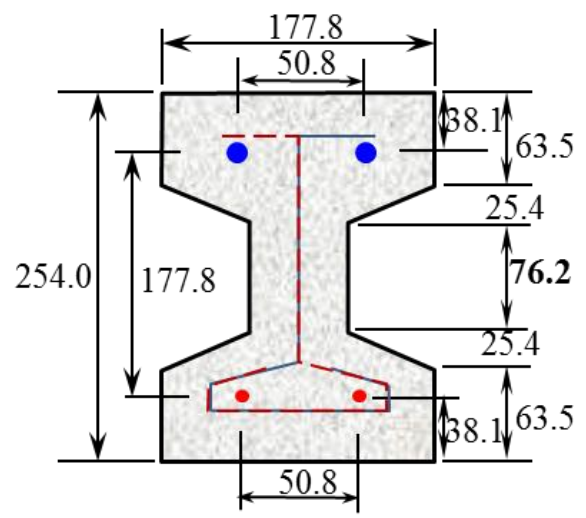

(a) Details of cross-section

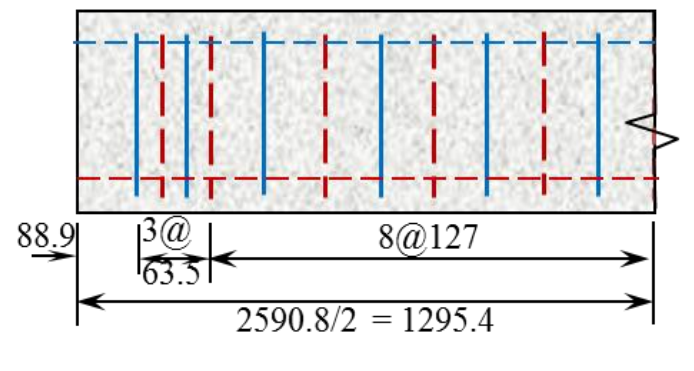

(b) Stirrups

Fig.5. Details of prestressed concrete test beam (mm) [14]. 


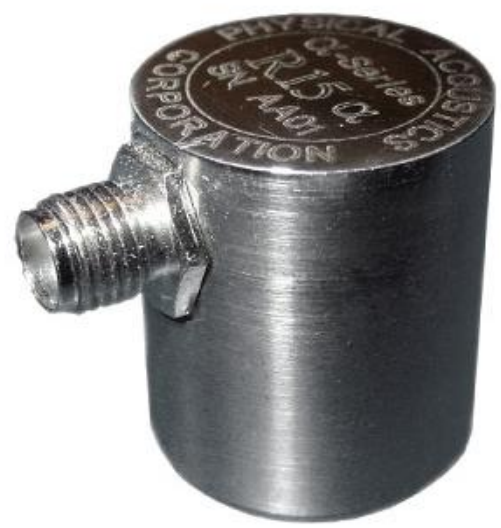

Fig.6. R15- $\alpha$ type AE sensor. 


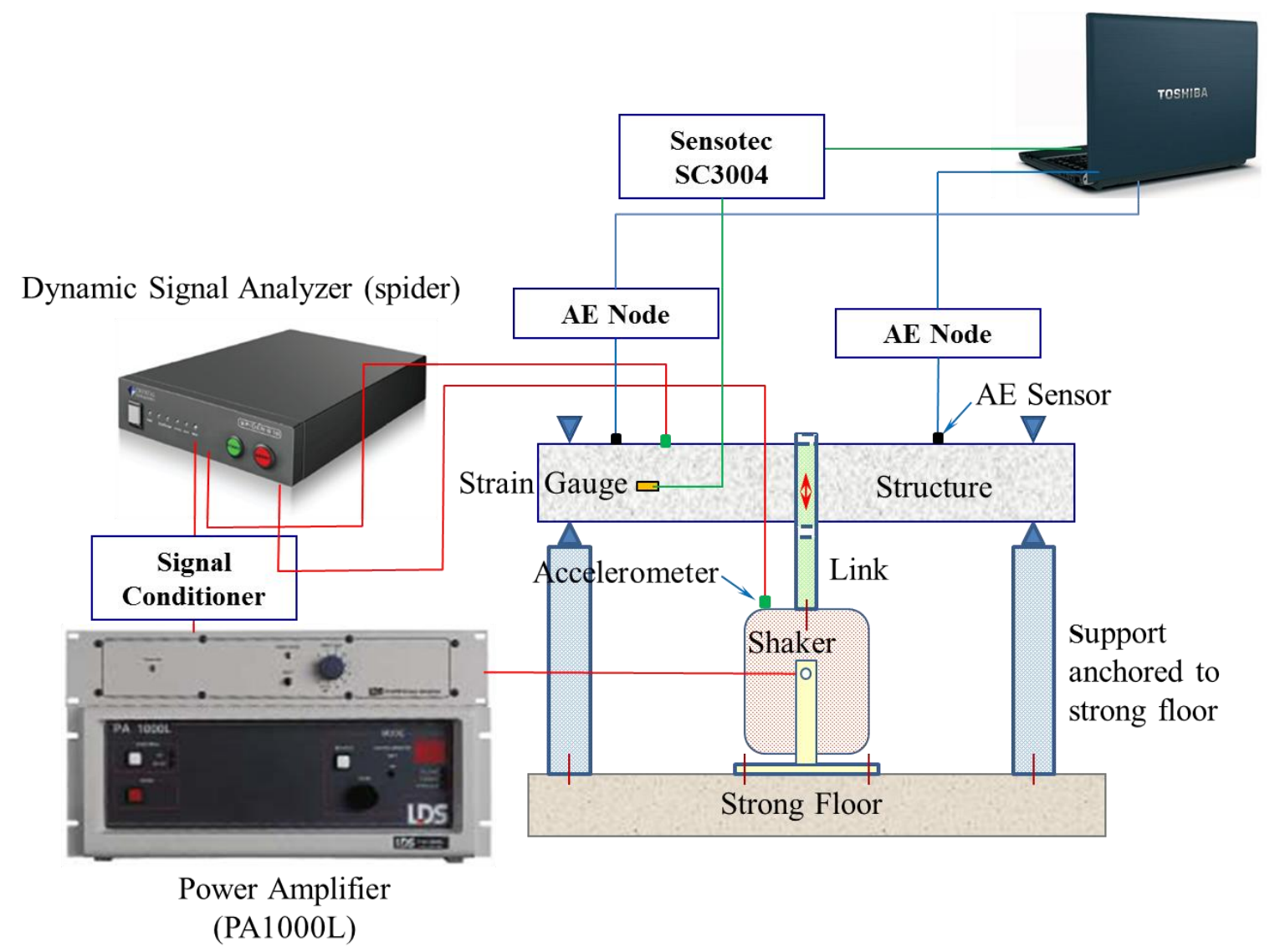

Fig.7. Test setup for reinforced concrete and steel beam specimens. 


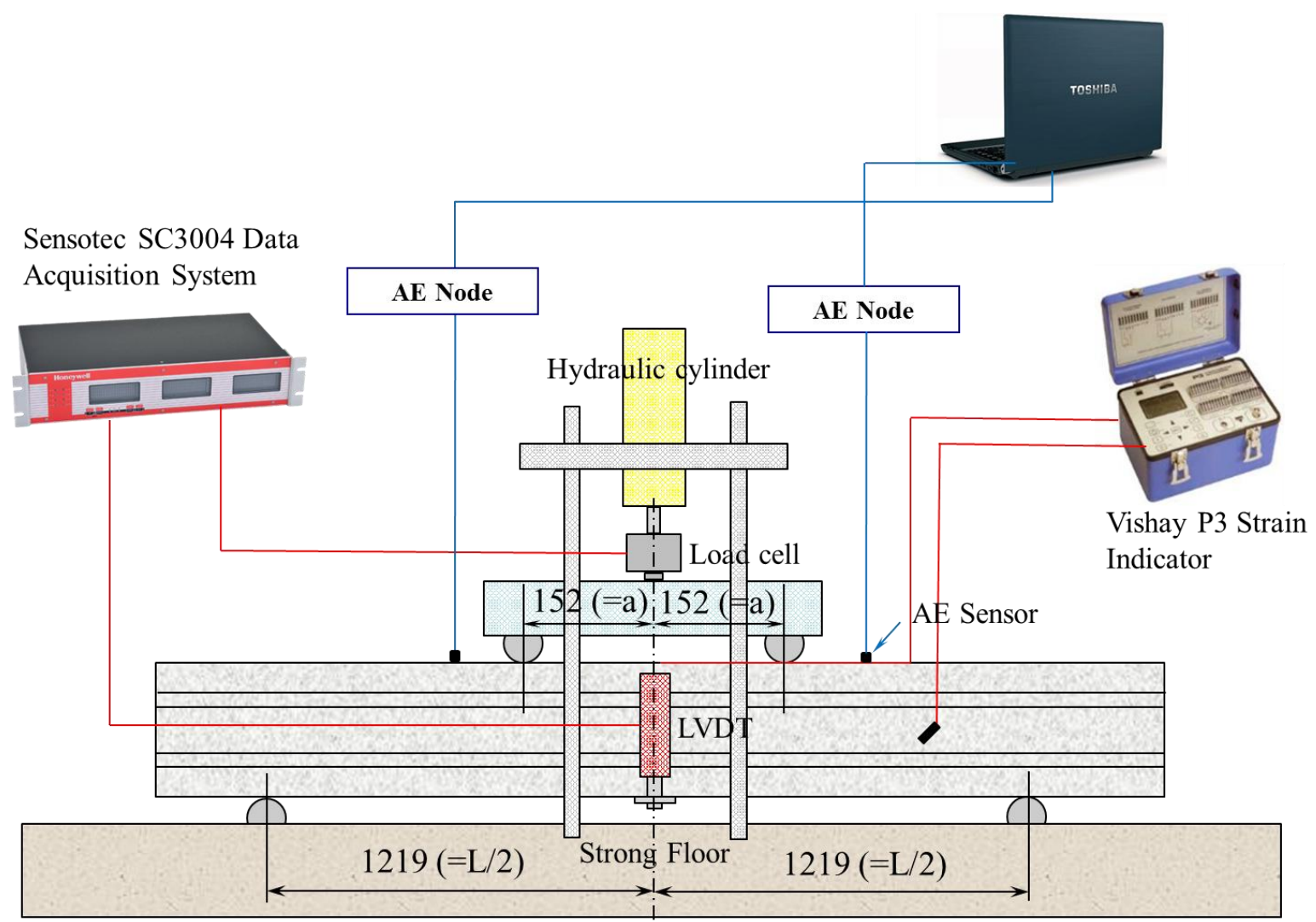

Fig.8. Test setup for prestressed concrete beam. 


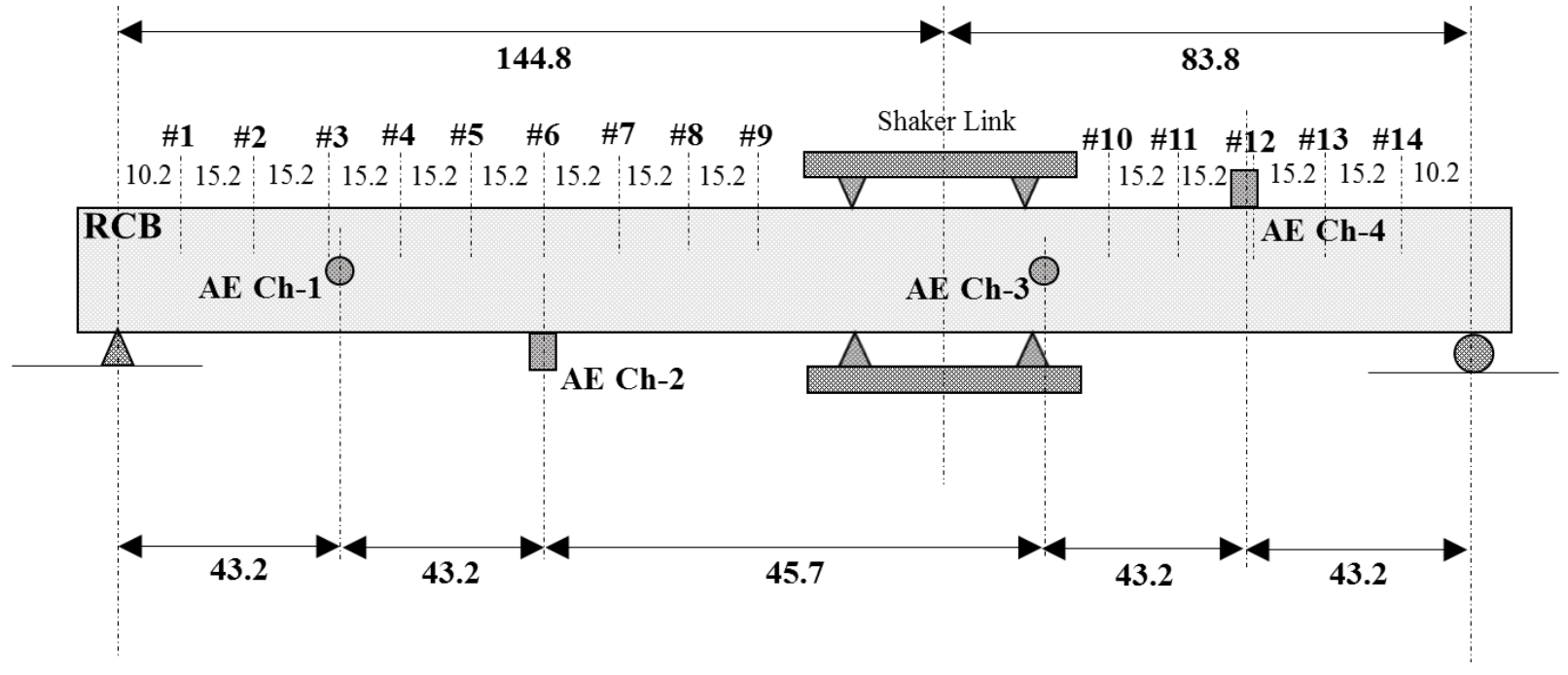

Fig.9. Training stations of reinforced concrete beam $(\mathrm{cm})$. 


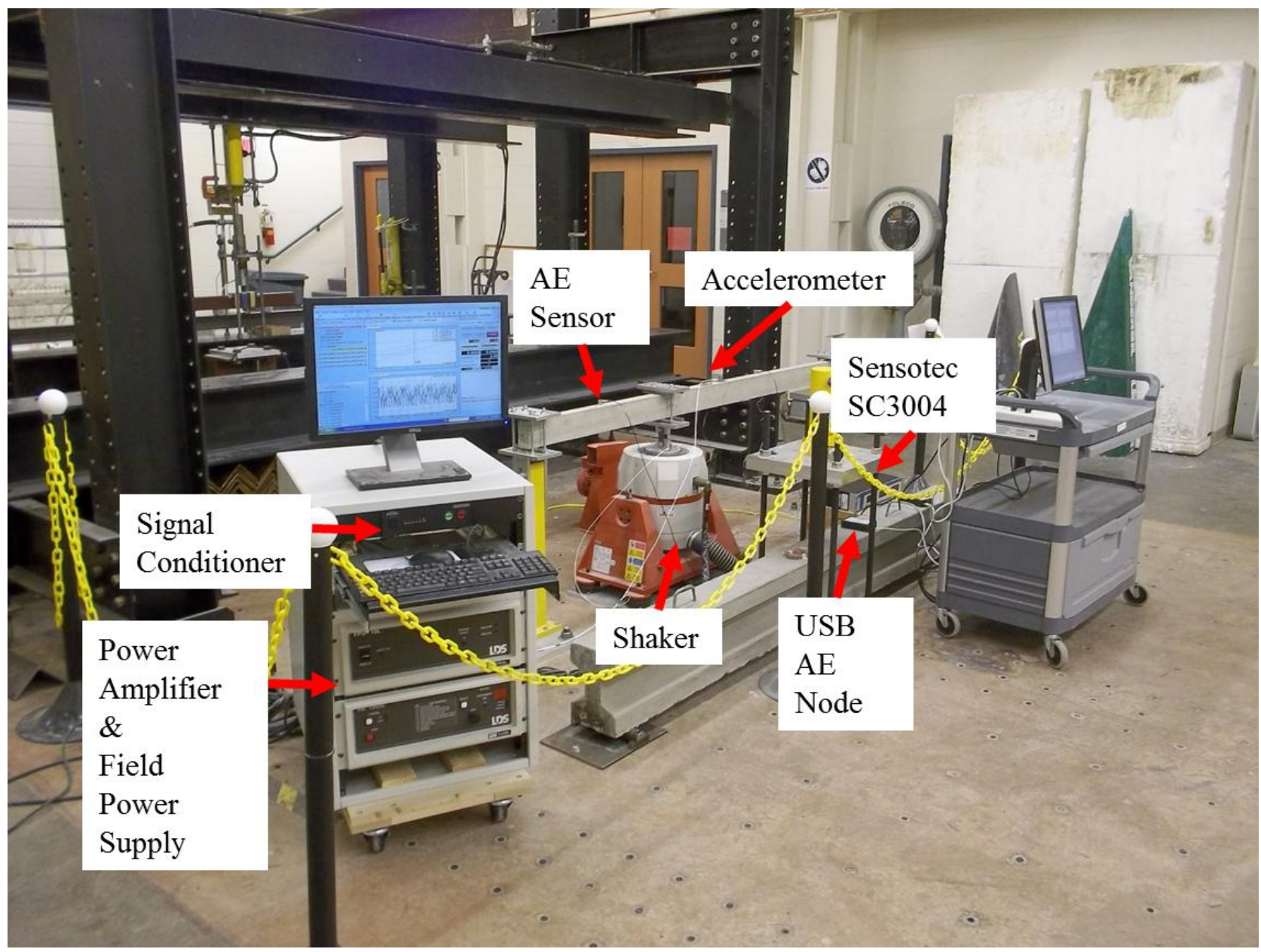

Fig.10. General test setup for reinforced concrete beam. 


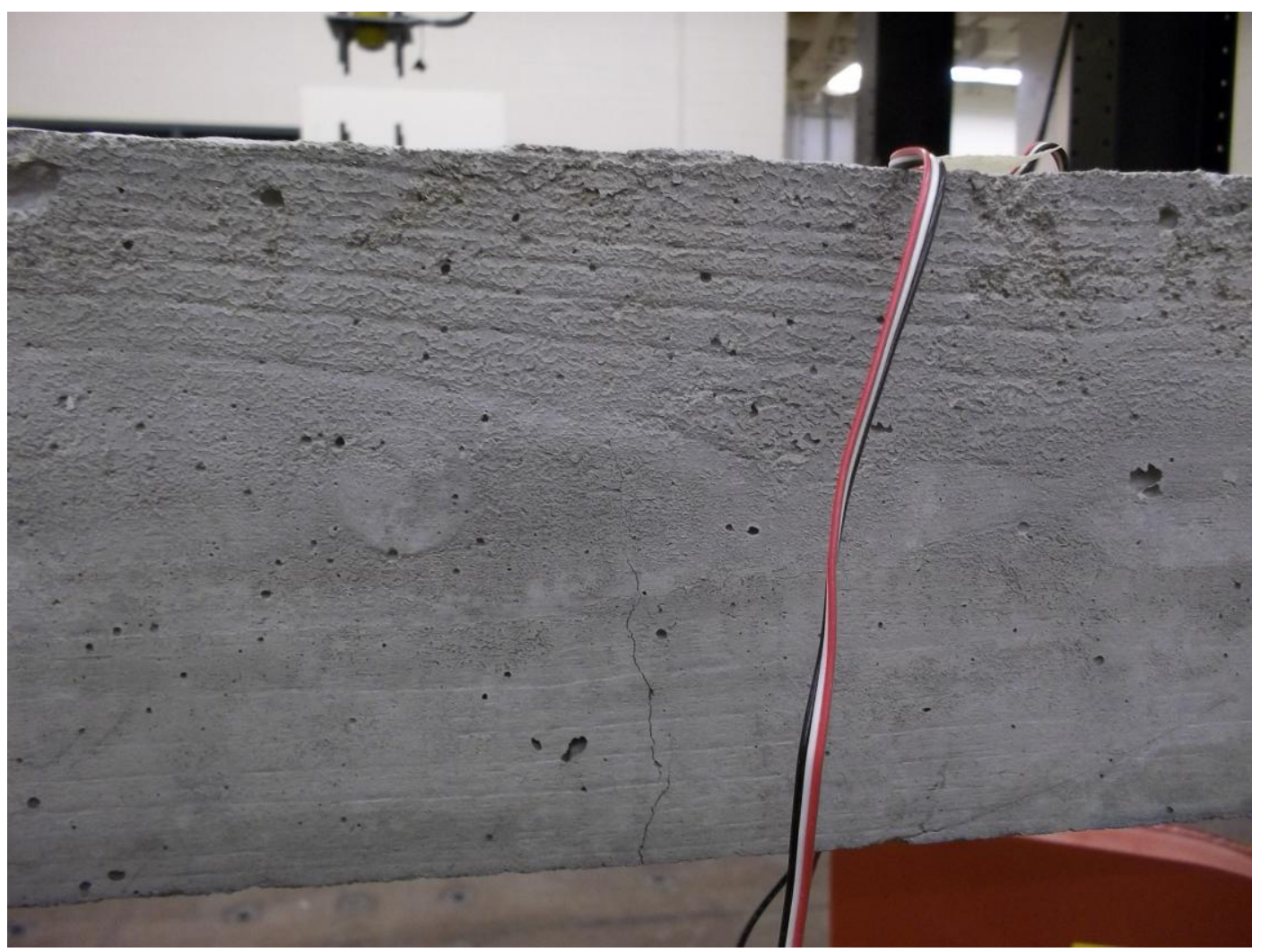

Fig.11. Crack in reinforced concrete beam in the longer segment 


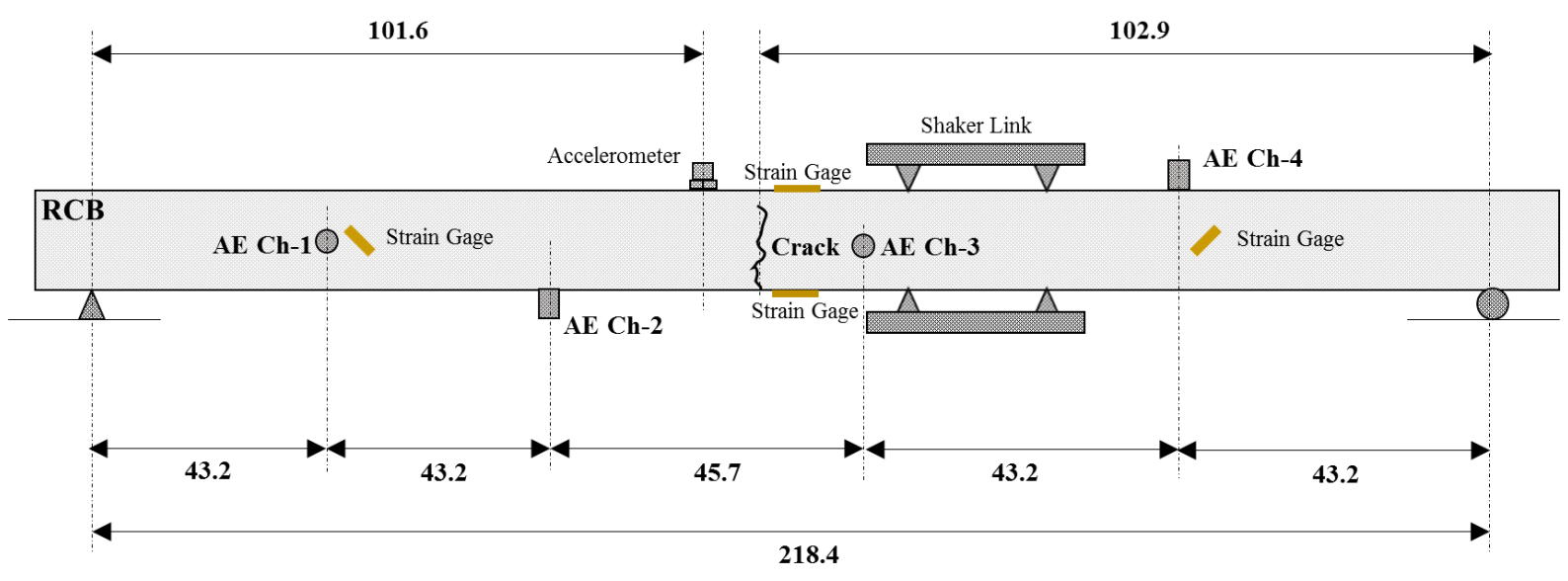

Fig.12. View of reinforced concrete beam showing the locations of the gages, sensors, accelerometer and crack $(\mathrm{cm})$. 


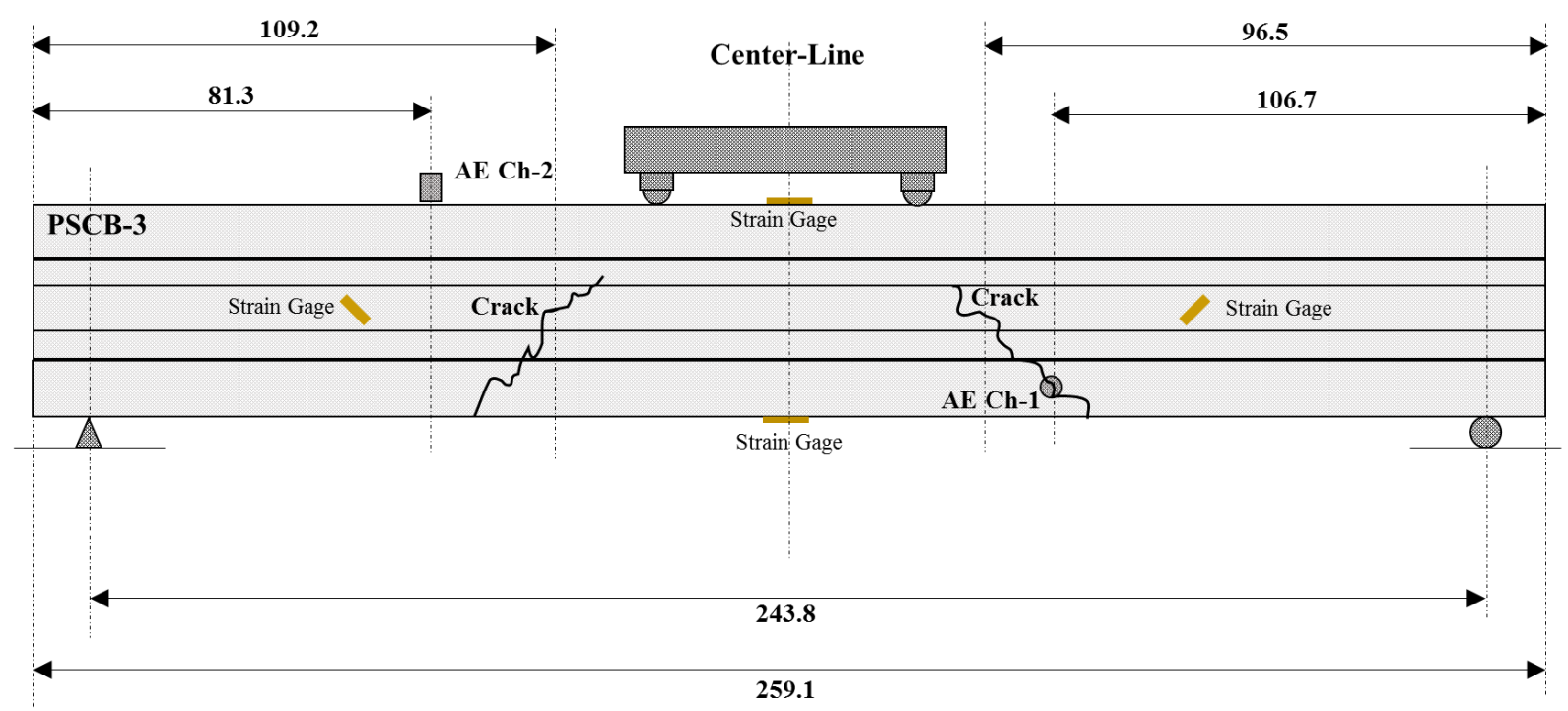

Fig.13. Location of gages, sensors and cracks after first loading cycle (cm). 


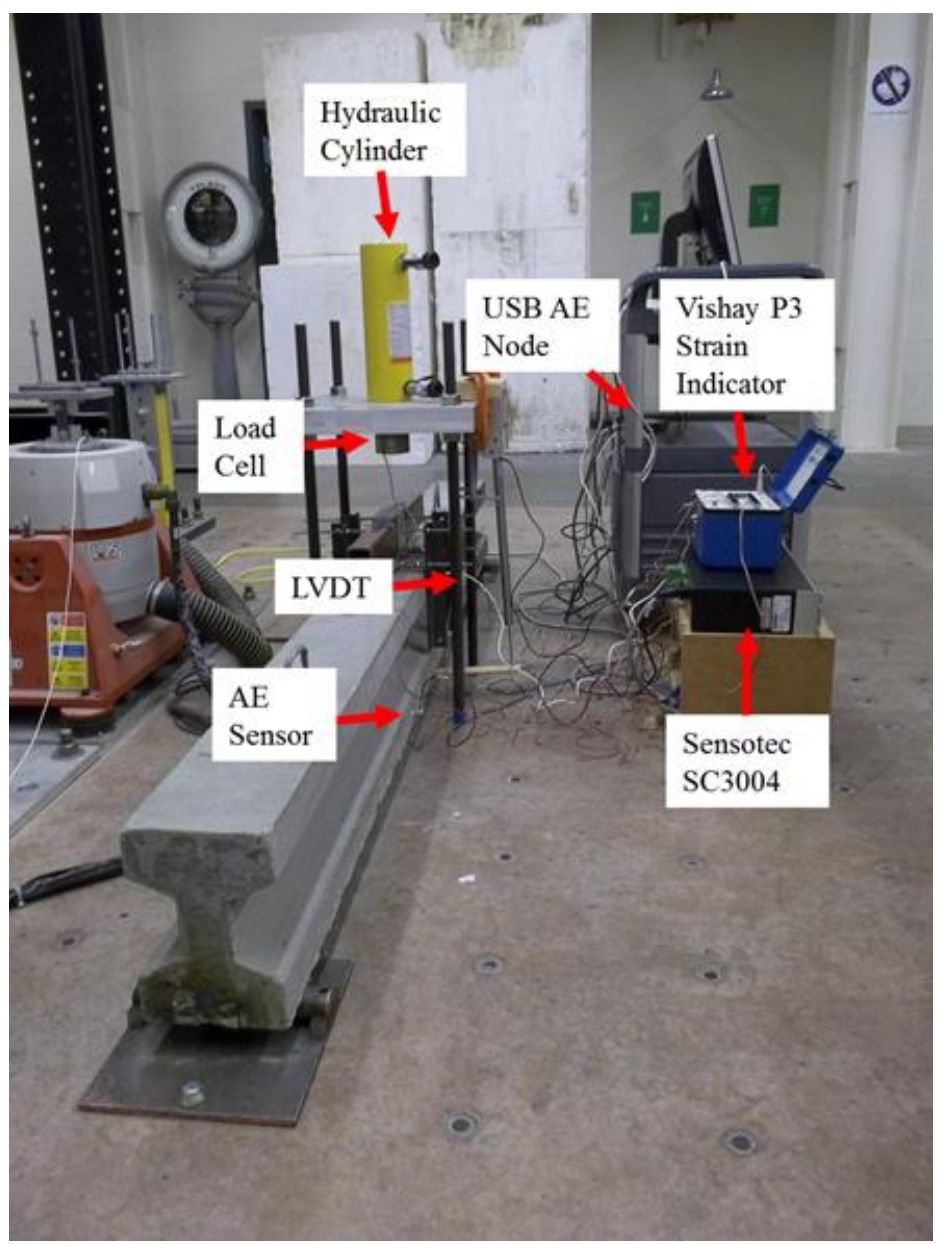

Fig.14. A view of test setup for prestressed concrete beam 

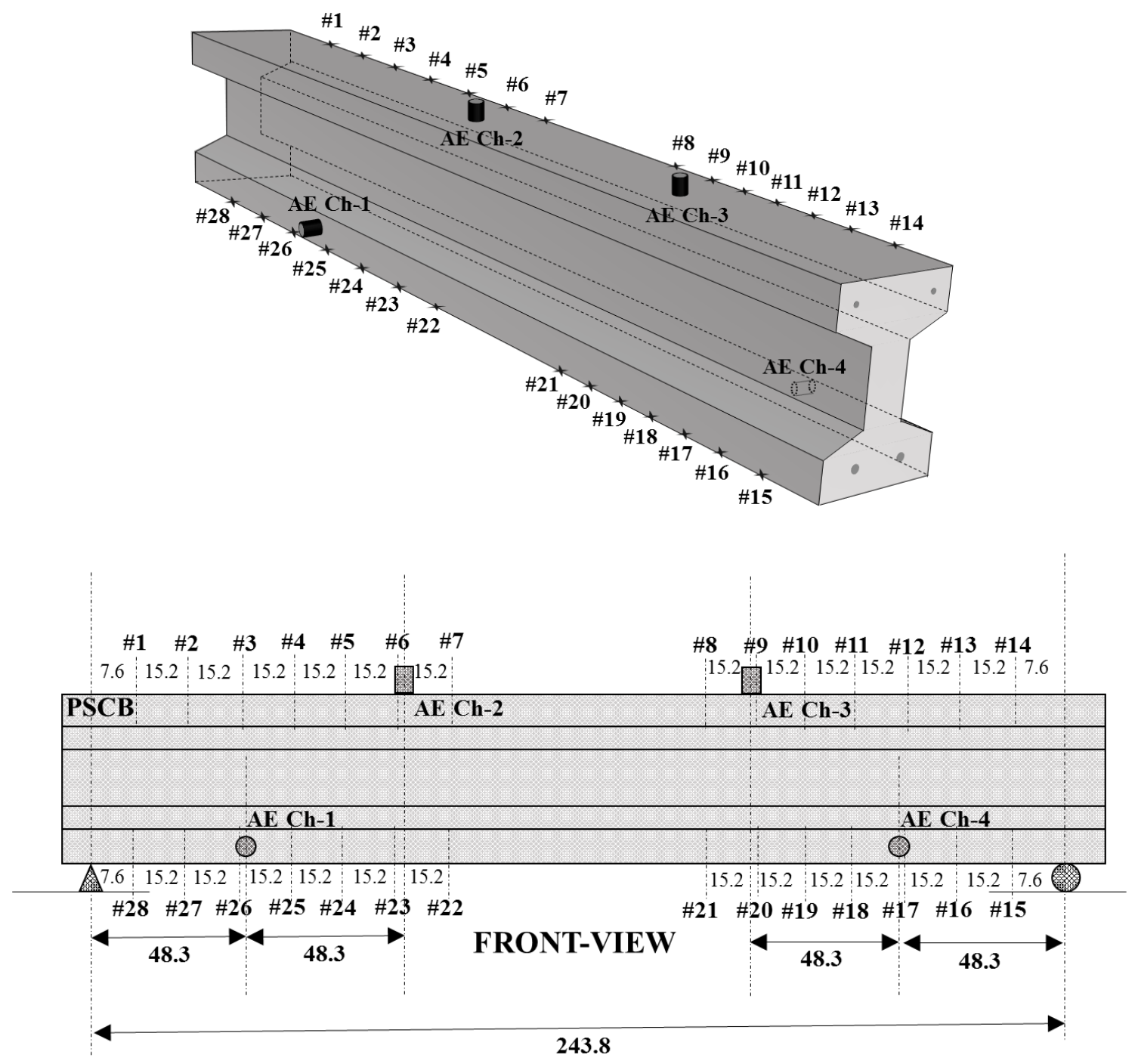

Fig.15. Training station locations for prestressed concrete beam $(\mathrm{cm})$. 


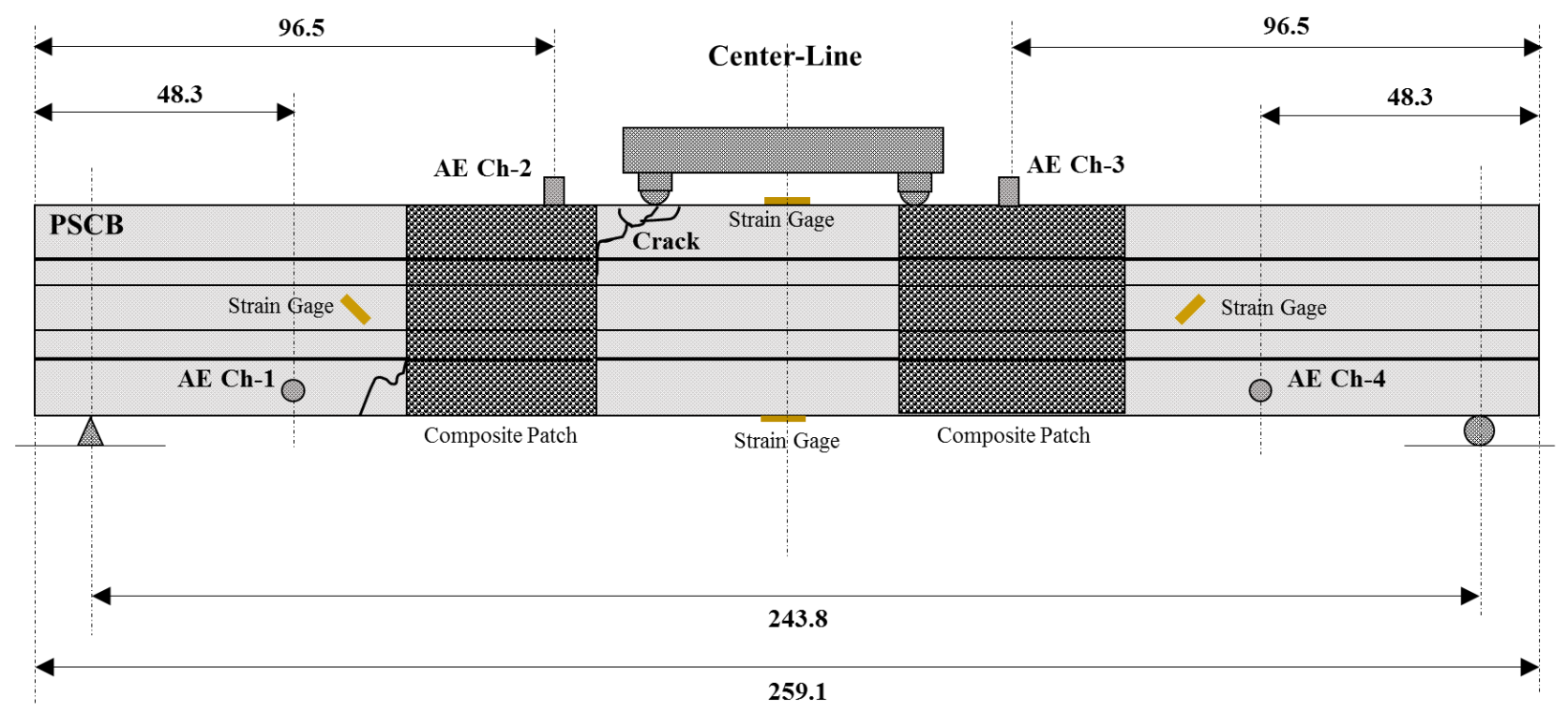

Fig.16. Locations of gages, sensors and cracks after repair (cm). 


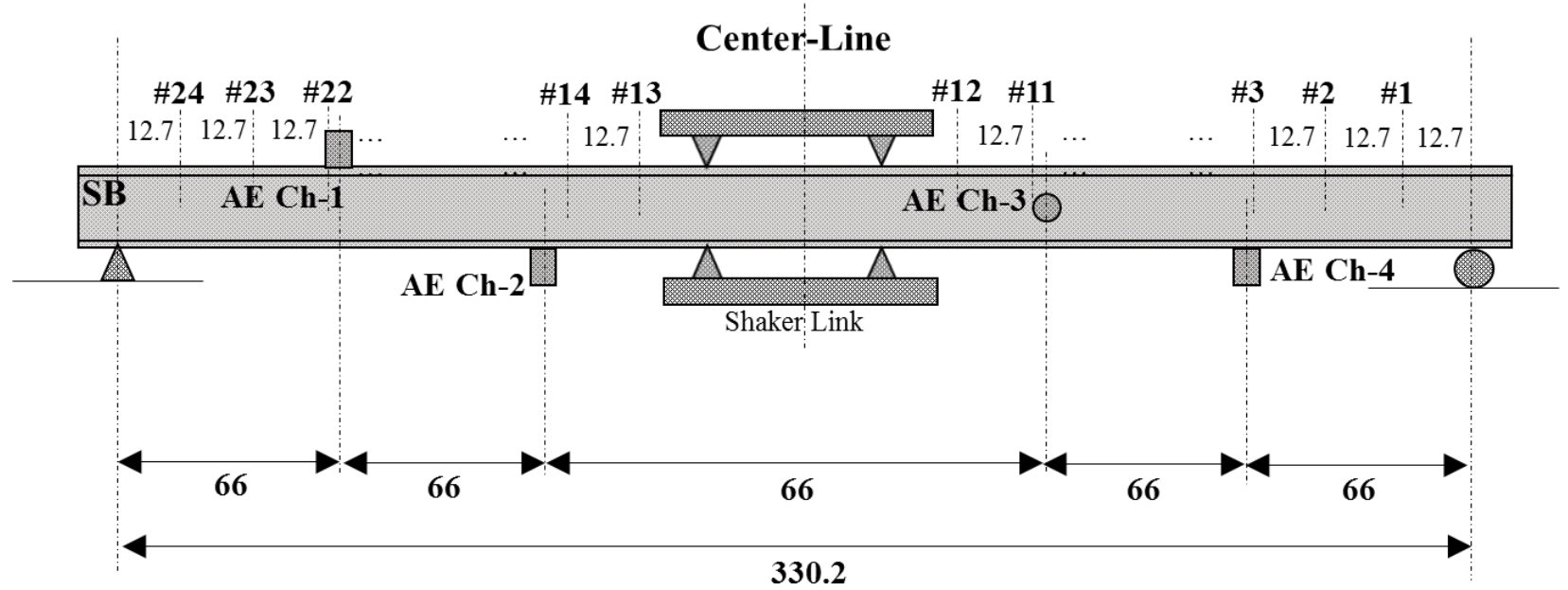

Fig.17. Training station locations for steel beam $(\mathrm{cm})$. 


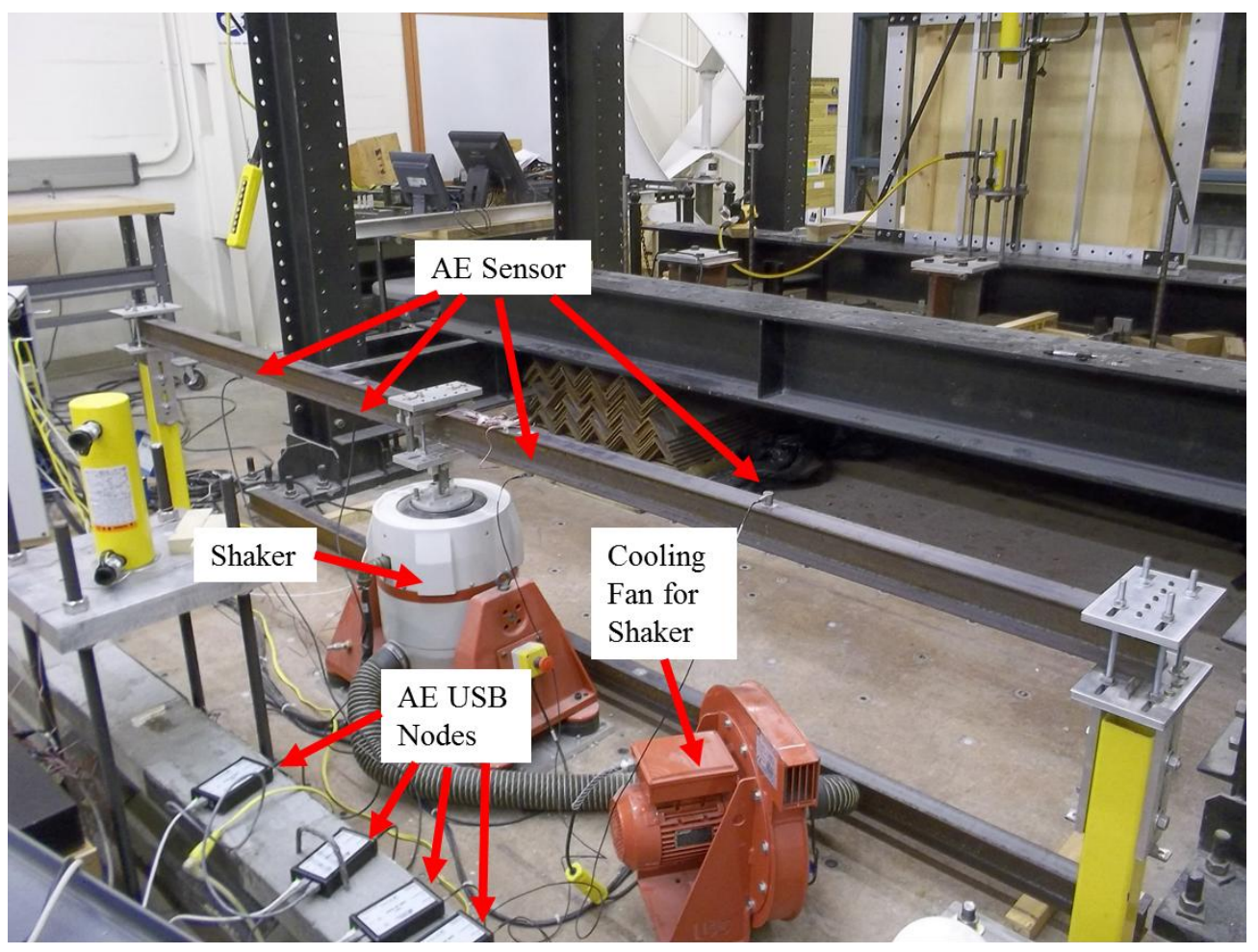

Fig. 18. General view of test setup for steel beam. 


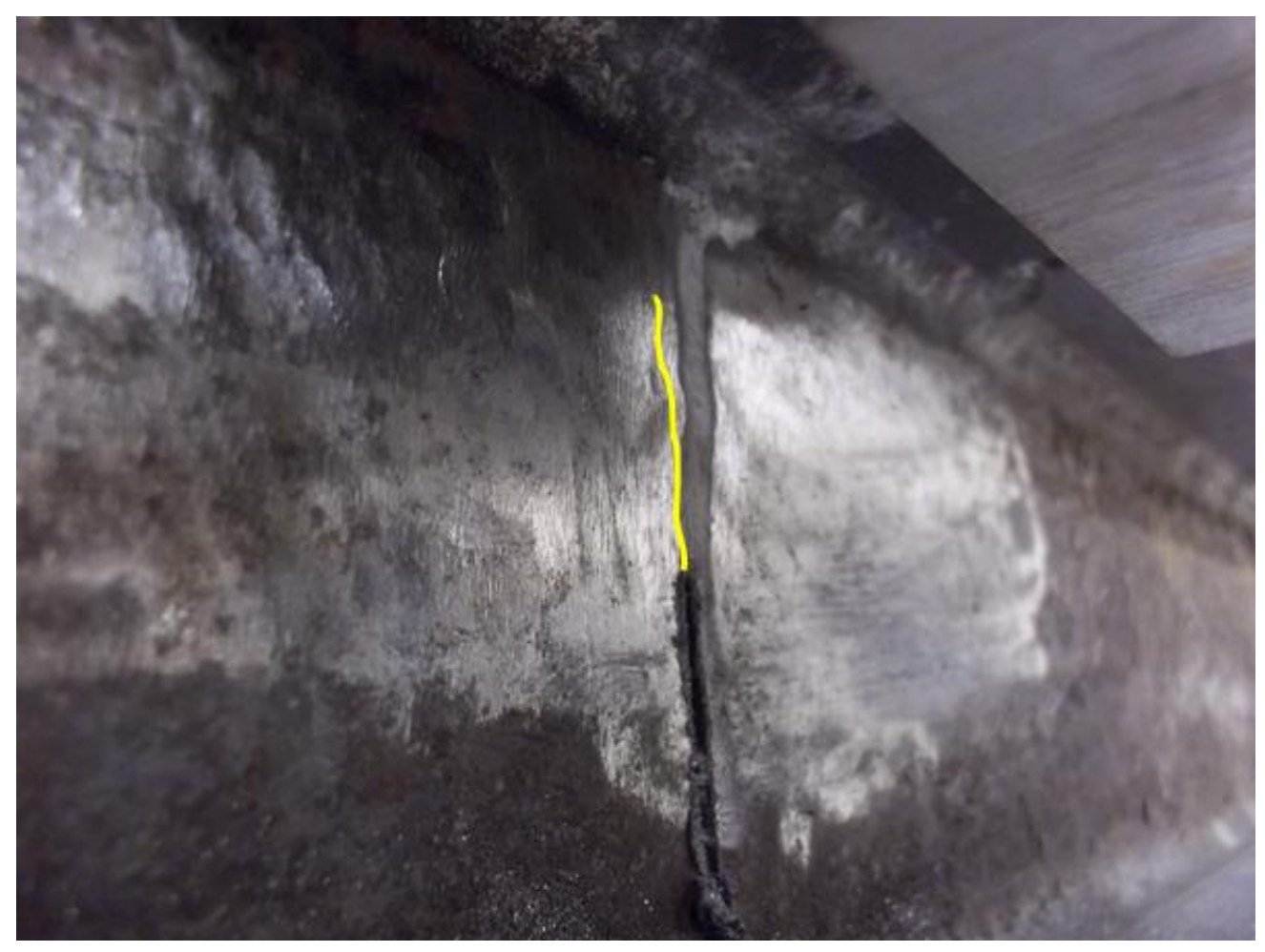

Fig. 19. Crack growth in steel beam (highlighted). 


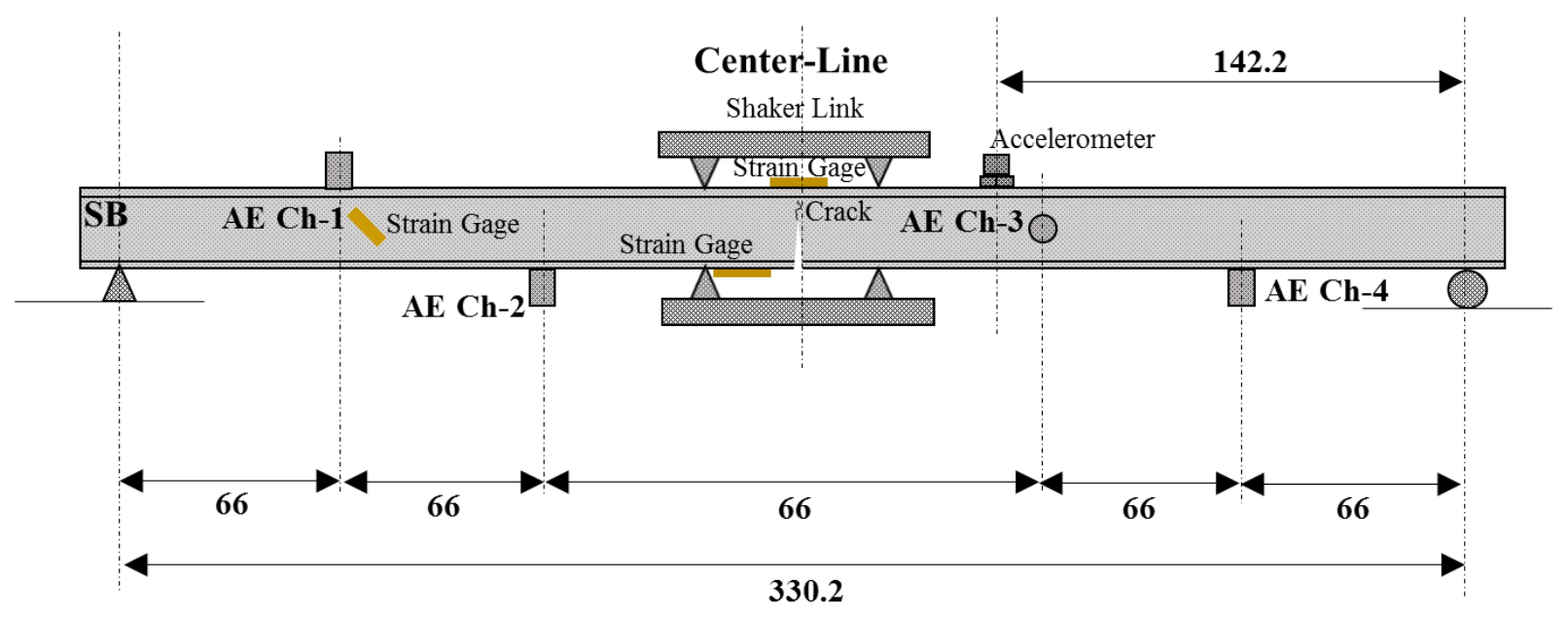

Fig. 20. Details of test setup for steel beam (cm). 


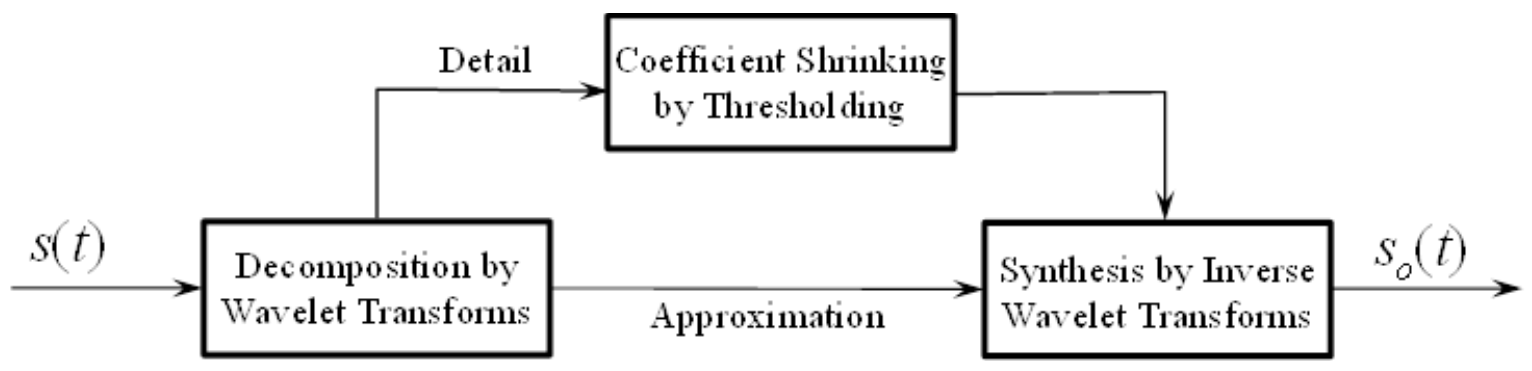

Fig.21. Denoising of signal 


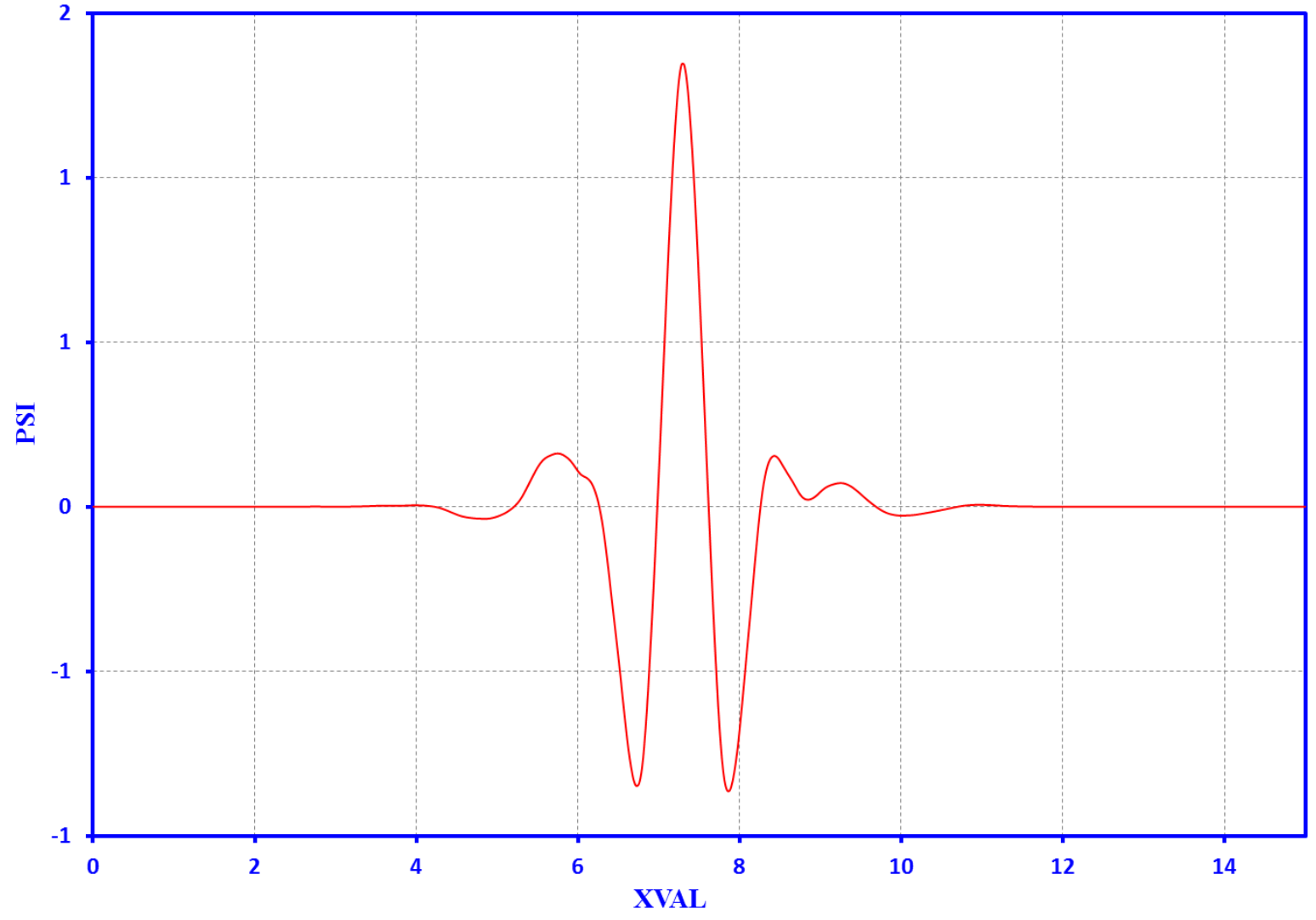

Figure 22. Mother Wavelet Sym8 


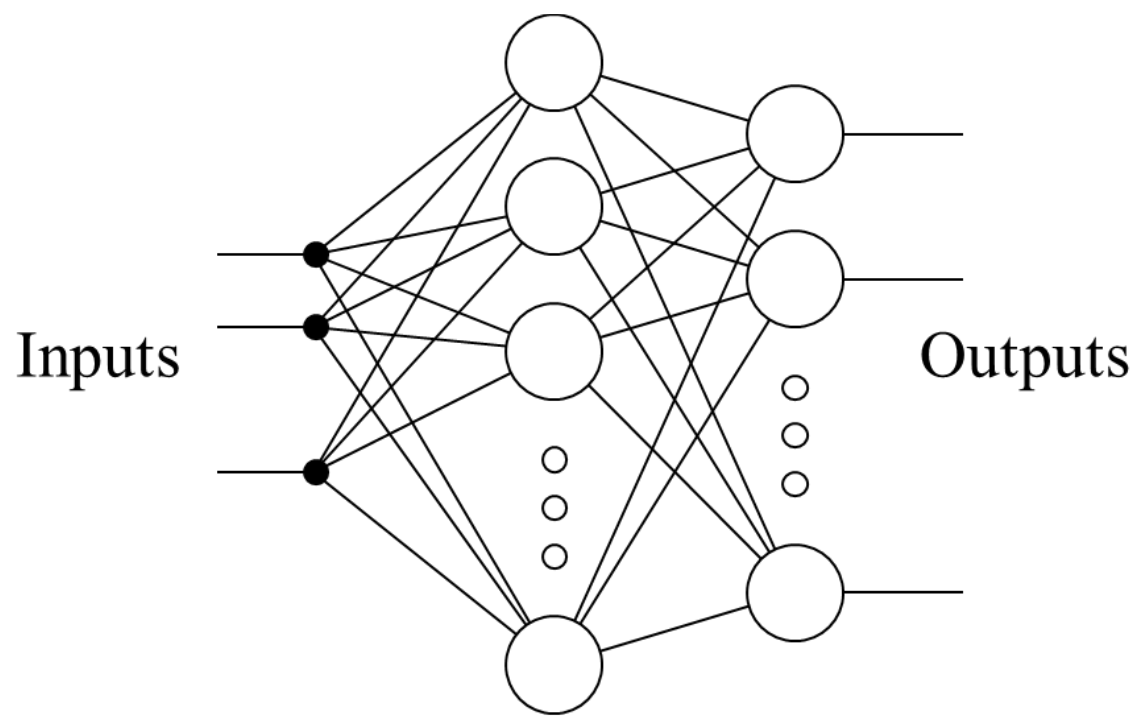

Hidden layer

Fig.23. Sample structure of a feed-forward neural network with one hidden layer. 

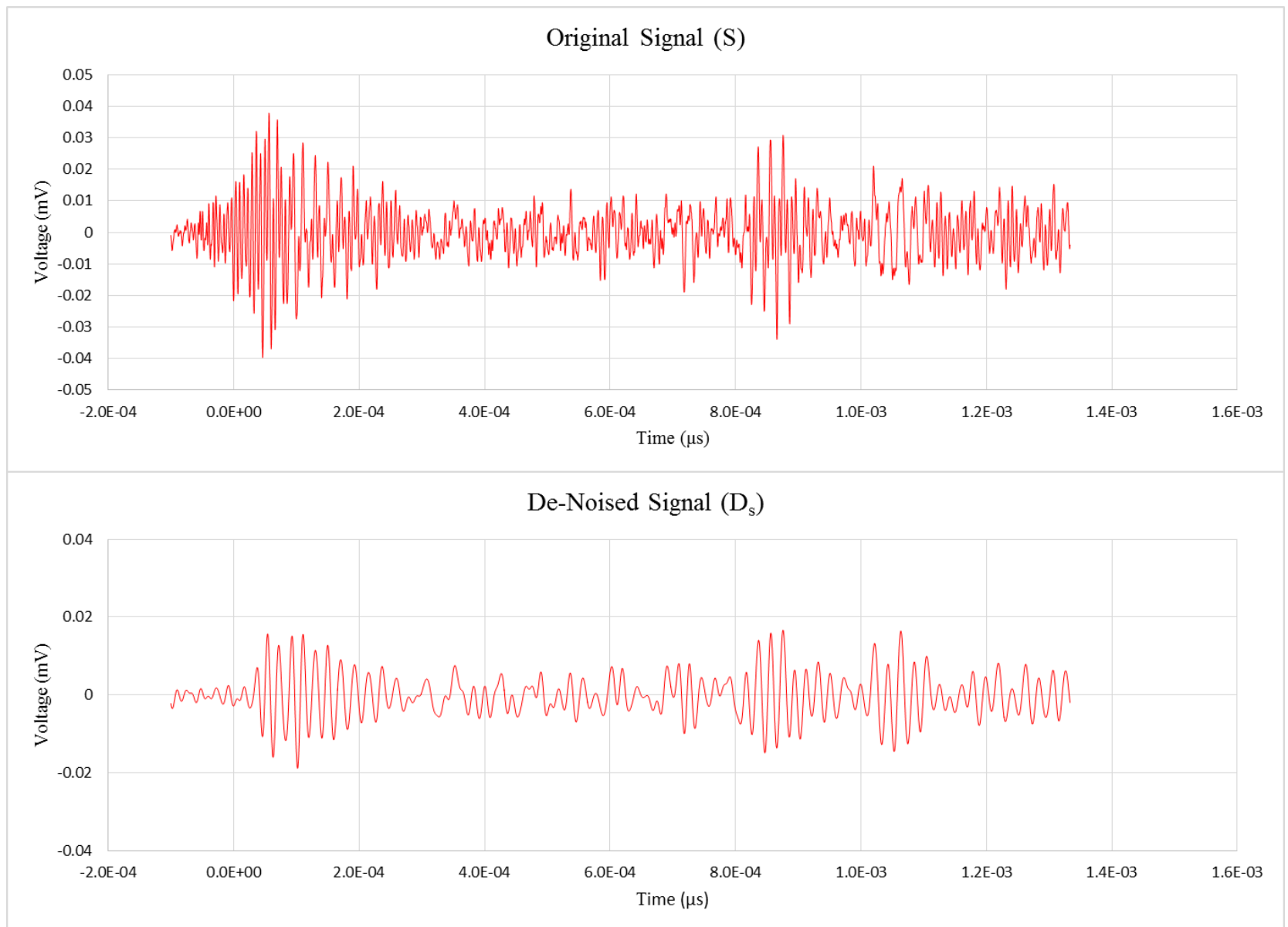

Fig. 24. Original and de-noised crack signals for the steel beam. 


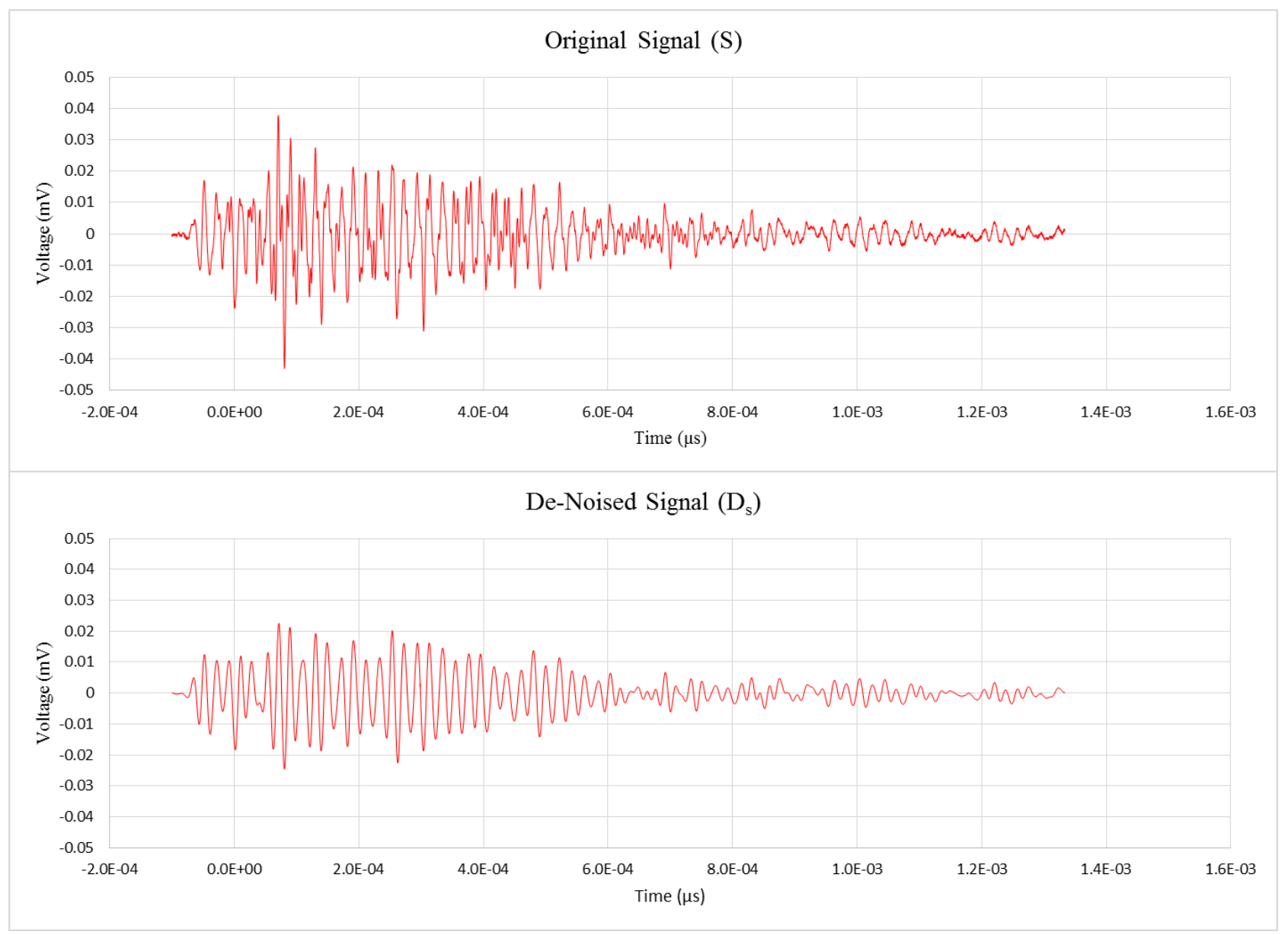

Fig. 25. Original and de-noised crack signals for the reinforced concrete beam. 


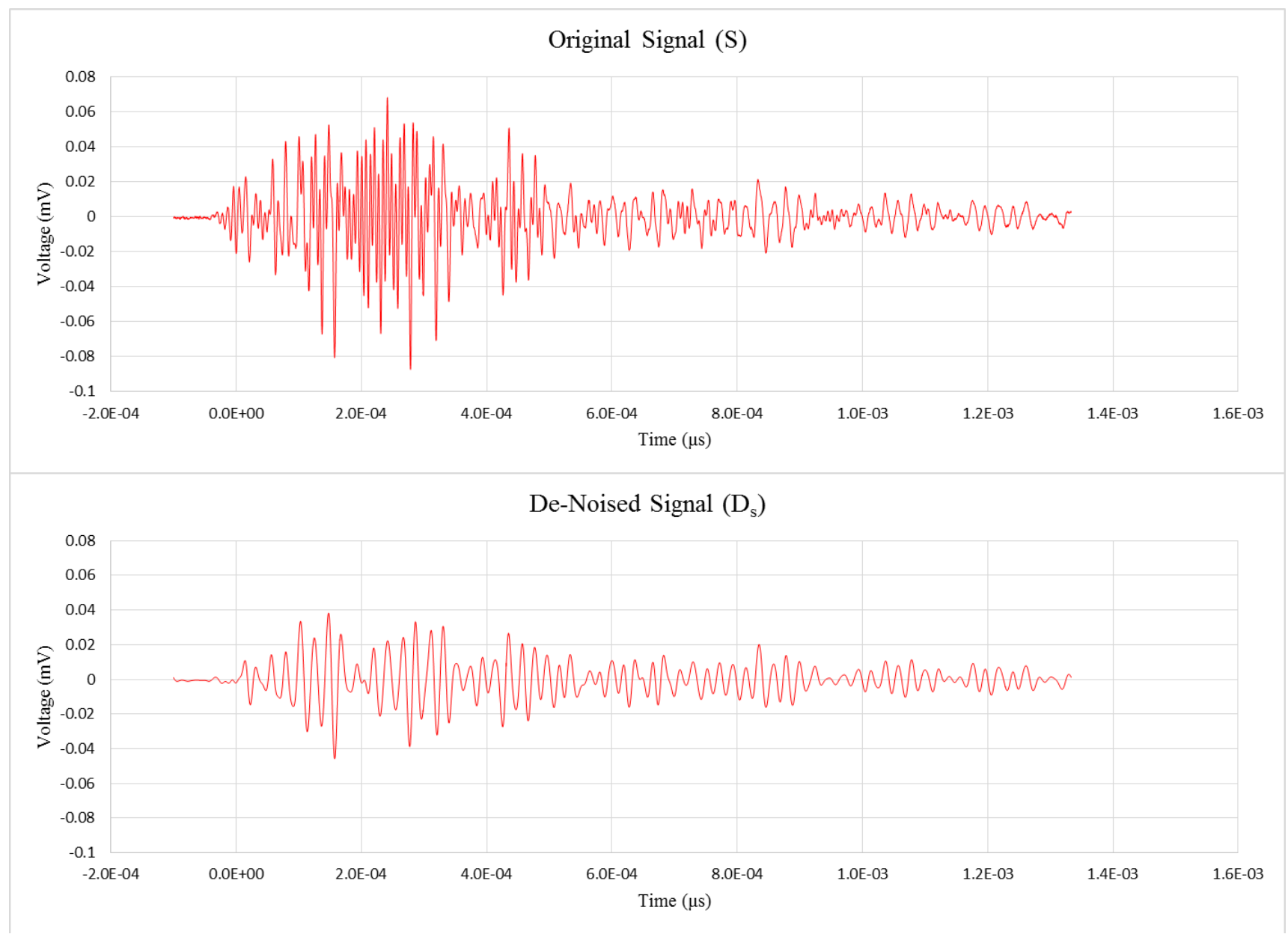

Fig. 26. Original and de-noised crack signals for the prestressed concrete beam. 


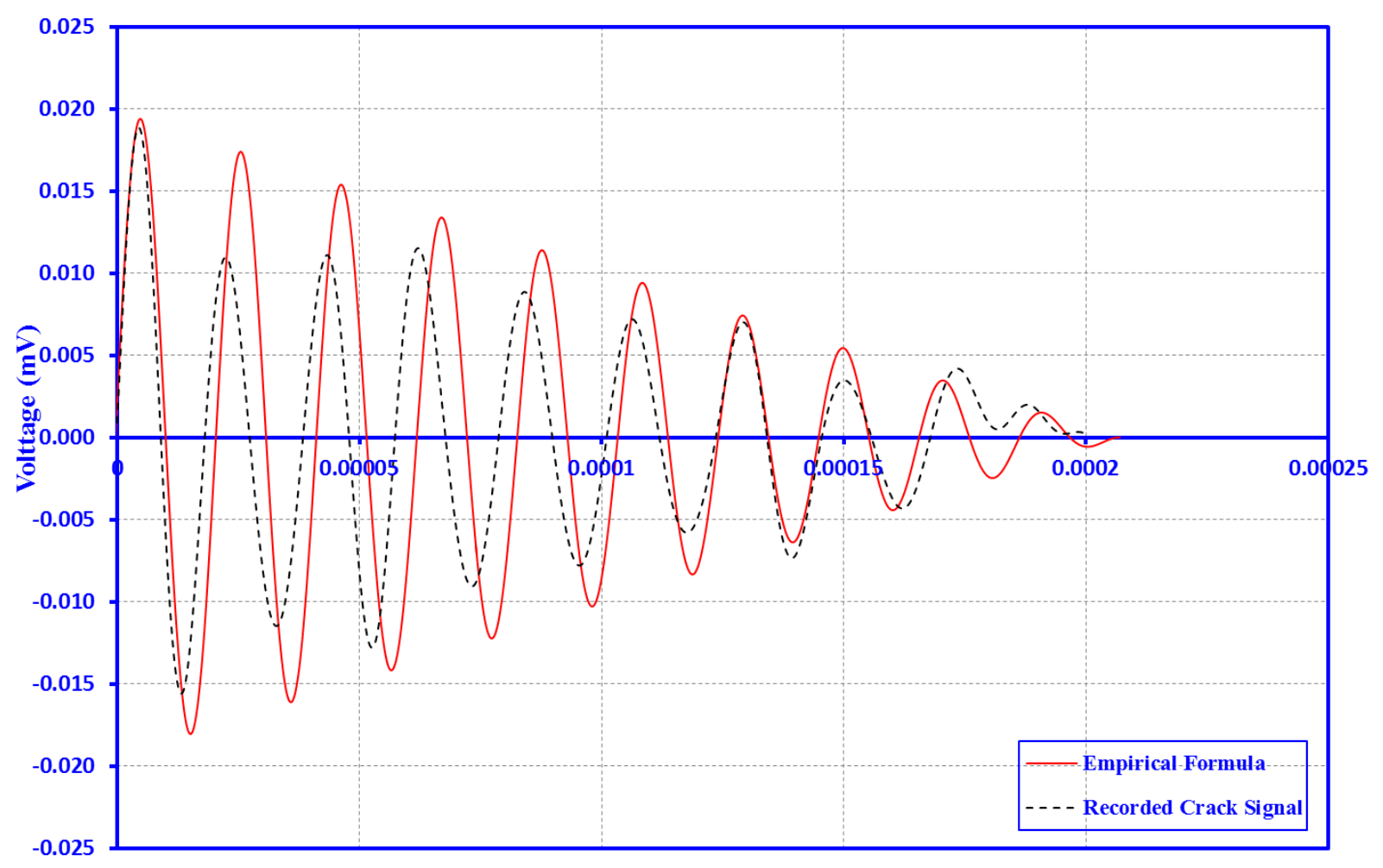

Time $(\mu \mathrm{s})$

Fig. 27. Comparison of empirical formula based acoustic signal with experimental data. 

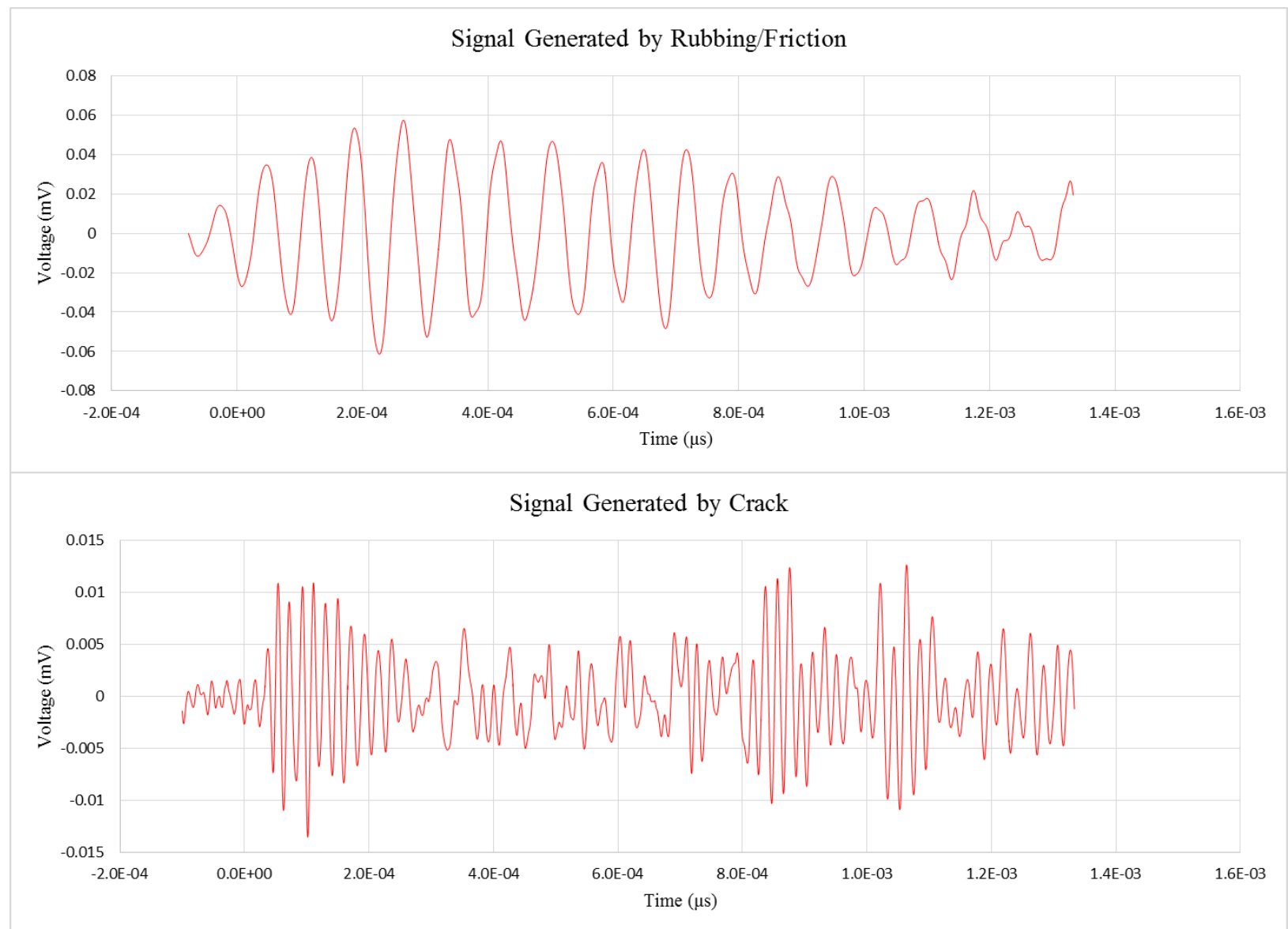

Fig.28. De-noised signals recorded from the steel beam originating from rubbing/friction (top plot) and from crack (bottom plot). 


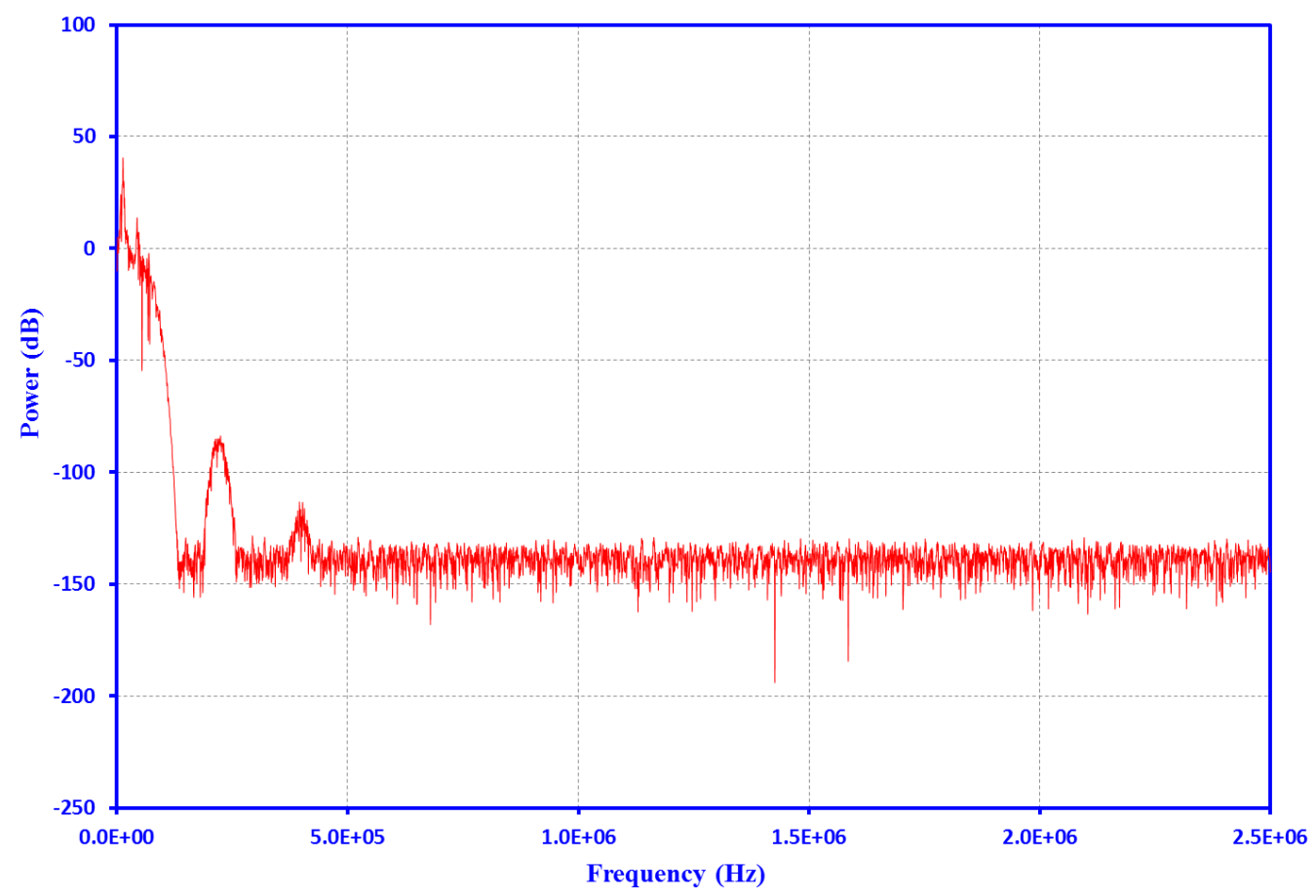

Fig.29. Power spectrum of the signal generated by rubbing/friction. 


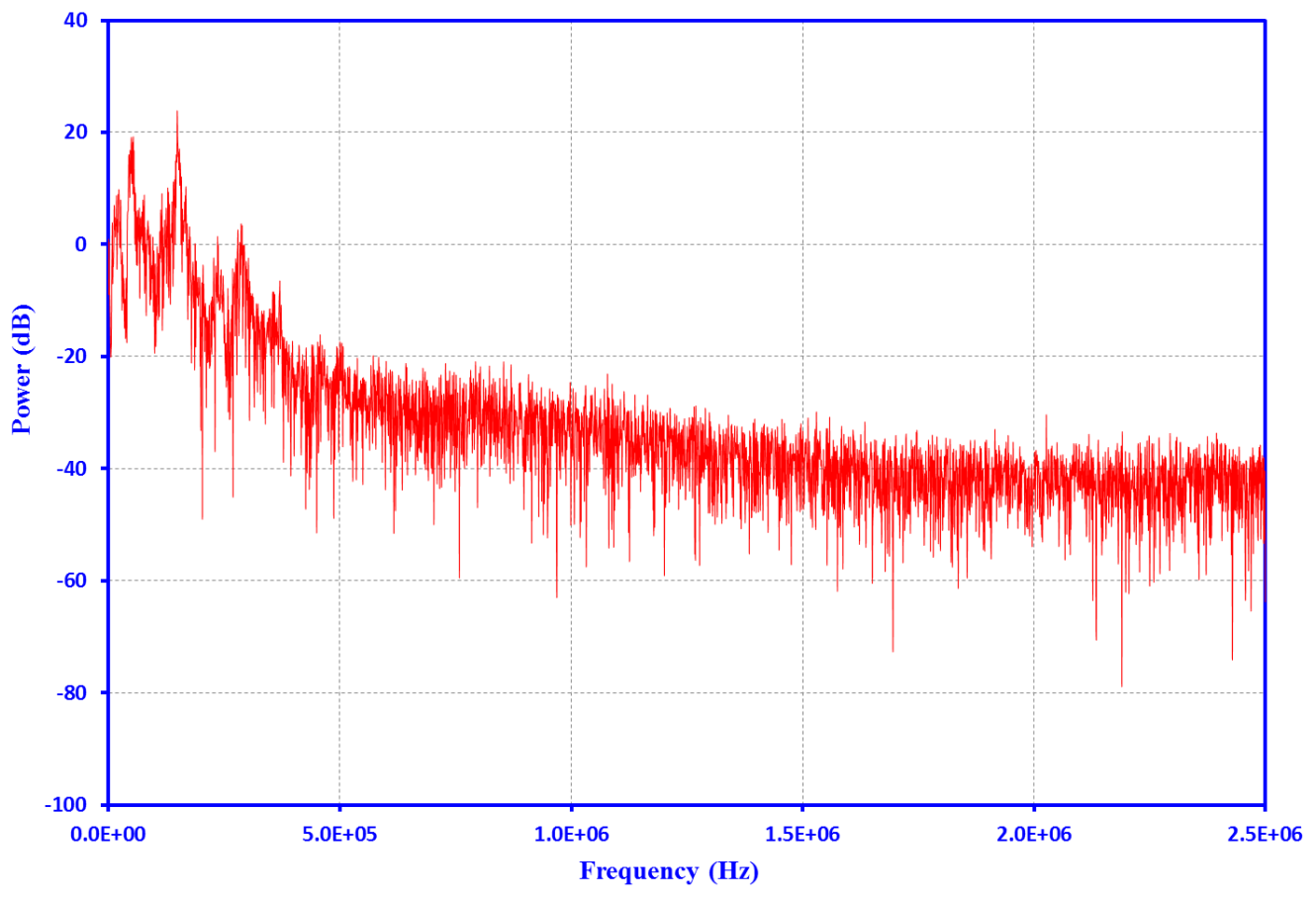

Fig.30. Power spectrum of the signal generated from crack. 

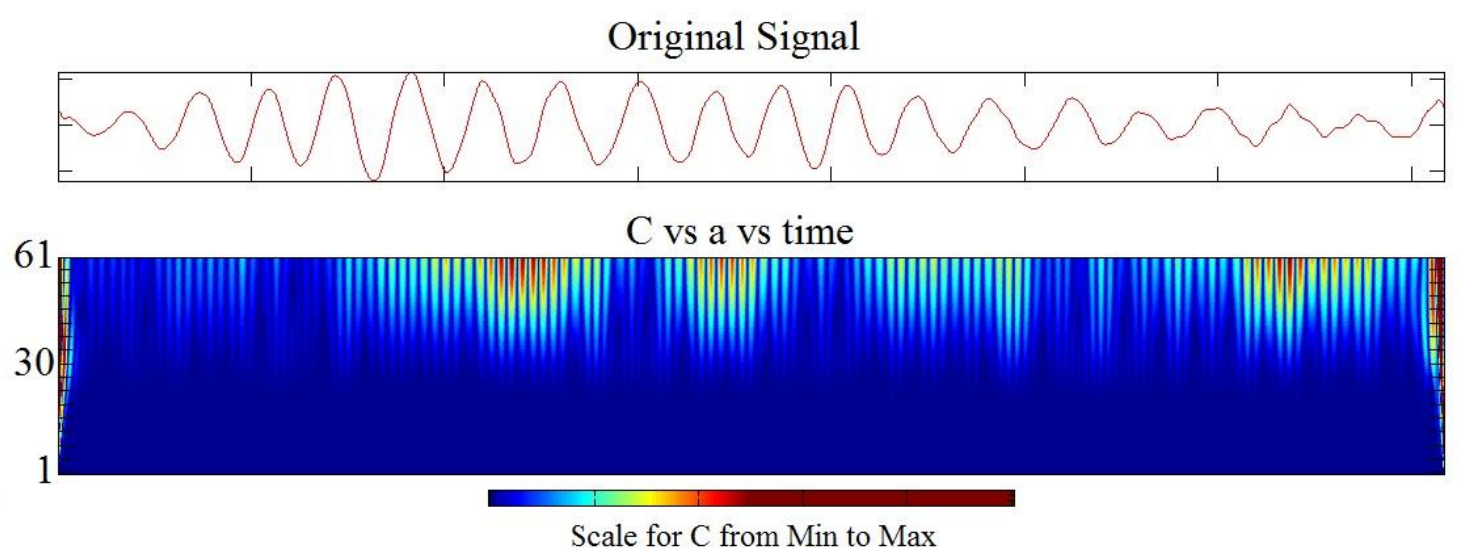

Fig.31. CWT of the signal generated by rubbing/friction. 


\section{Original Signal}
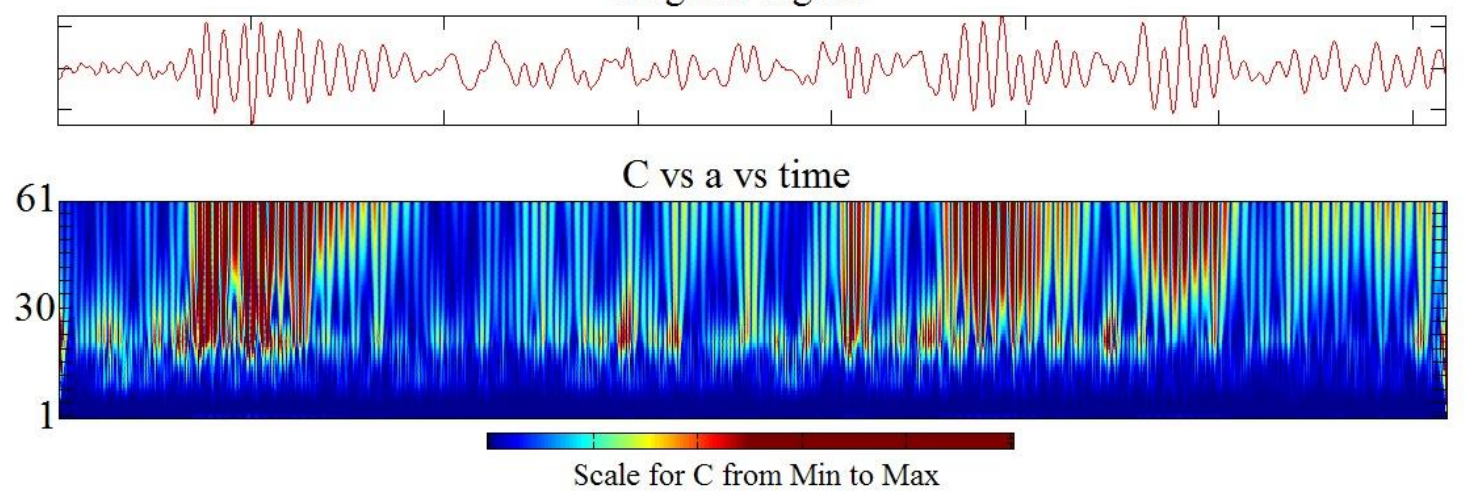

Fig.32. CWT of the signal generated by crack. 
Table-1. S3x5.7 properties from 2010 AISC Steel Manual [15]

\begin{tabular}{|lrllrl|}
\hline $\mathrm{A}=$ & 1071 & $\mathrm{~mm}^{2}$ & $\mathrm{k}=$ & 13.5 & $\mathrm{~mm}$ \\
$\mathrm{~d}=$ & 76.2 & $\mathrm{~mm}$ & $\mathrm{~T}=$ & 44.45 & $\mathrm{~mm}$ \\
$\mathrm{t}_{\mathrm{w}}=$ & 4.3 & $\mathrm{~mm}$ & $\mathrm{I}_{\mathrm{x}}=$ & $10.4 \mathrm{e} 5$ & $\mathrm{~mm}^{4}$ \\
$\mathrm{~b}_{\mathrm{f}}=$ & 59.2 & $\mathrm{~mm}$ & $\mathrm{I}_{\mathrm{y}}=$ & $18.6 \mathrm{e} 4$ & $\mathrm{~mm}^{4}$ \\
$\mathrm{t}_{\mathrm{f}}=$ & 6.6 & $\mathrm{~mm}$ & & & \\
\hline
\end{tabular}


Table-2. Specifications of the R15- $\alpha$ sensor

\begin{tabular}{ll}
\hline Parameter & Corresponding value \\
\hline Operating Frequency Range & $50-400 \mathrm{kHz}$ \\
Resonant Frequency & $75 \mathrm{kHz}$ \\
Peak Sensitivity & $80 \mathrm{~dB}$ \\
Dimensions & $19.05 \mathrm{~mm}($ dia. $) \times 22.35 \mathrm{~mm}(\mathrm{~h})$ \\
\hline
\end{tabular}


Table-3. Performance parameters of the shaker

\begin{tabular}{ll}
\hline Parameter & Corresponding value \\
\hline Sine Force (Peak) & $165 \mathrm{~kg}$ \\
Armature Resonance $\left(\mathrm{f}_{\mathrm{n}}\right)$ & $3.8 \mathrm{kHz}$ \\
Usable Frequency Range & $5 \mathrm{~Hz}-4 \mathrm{kHz}$ \\
Velocity (sine peak) & $1397 \mathrm{~mm} / \mathrm{sec}$ \\
Acceleration (sine peak) & $73.7 \mathrm{~g}$ \\
Displacement (peak-peak) & $25.4 \mathrm{~mm}$ \\
\hline
\end{tabular}


Table-4. Comparison of actual crack location in the steel beam with neural network predictions (Distances are from left end)

\begin{tabular}{lll}
\hline Neural Network prediction & Actual crack location & Error \\
\hline $1618.42 \mathrm{~mm}$ & $1651.00 \mathrm{~mm}$ & $0.99 \%$ \\
$1610.67 \mathrm{~mm}$ & $1651.00 \mathrm{~mm}$ & $1.22 \%$ \\
$1469.52 \mathrm{~mm}$ & $1651.00 \mathrm{~mm}$ & $5.49 \%$ \\
$1649.59 \mathrm{~mm}$ & $1651.00 \mathrm{~mm}$ & $0.04 \%$ \\
$1720.52 \mathrm{~mm}$ & $1651.00 \mathrm{~mm}$ & $2.11 \%$ \\
\hline
\end{tabular}


Table-5. Comparison of actual crack location in the reinforced concrete with neural network predictions (Distances are from left end)

\begin{tabular}{lll}
\hline Neural Network prediction & Actual crack location & Error \\
\hline $993.91 \mathrm{~mm}$ & $1028.70 \mathrm{~mm}$ & $3.38 \%$ \\
$1034.45 \mathrm{~mm}$ & $1028.70 \mathrm{~mm}$ & $0.56 \%$ \\
$1005.77 \mathrm{~mm}$ & $1028.70 \mathrm{~mm}$ & $2.23 \%$ \\
$1034.06 \mathrm{~mm}$ & $1028.70 \mathrm{~mm}$ & $0.52 \%$ \\
$1016.51 \mathrm{~mm}$ & $1028.70 \mathrm{~mm}$ & $1.18 \%$ \\
\hline
\end{tabular}

